\title{
HYPERSURFACES OF ORDER TWO
}

\section{BY}

\section{TIBOR BISZTRICZKY}

ABSTRACT. A hypersurface $S^{n-1}$ of order two in the real projective $n$-space is met by every straight line in maximally two points; cf. [1, p. 391]. We develop a synthetic theory of these hypersurfaces inductively, basing it upon a concept of differentiability. We define the index and the degree of degeneracy of an $S^{n-1}$ and classify the $S^{n-1}$ in terms of these two quantities. Our main results are (i) the reduction of the theory of the $s^{n-1}$ to the nondegenerate case; (ii) the Theorem (A.5.11) that a nondegenerate $S^{n-1}$ of positive index must be a quadric and (iii) a comparison of our theory with Marchaud's discussion of "linearly connected" sets; cf. [3].

Preface. The theory of plane curves is of major importance in our study of the hypersurfaces of order two. This theory is the first step in our induction and the means by which we define tangents. The introduction of these curves follows the approach of P. Scherk in [5] and R. Park in [4] .

A. Marchaud introduced in [2] the "surfaces of order two" in the real projective three-space. Our theory is based on that paper.

We compare our hypersurfaces with the quadrics by direct construction (see Appendix) and also by showing that these hypersurfaces are identical with the common boundaries of certain pairs of linearly connected sets; cf. [3] .

This paper has developed out my doctoral thesis of the same title, written at the University of Toronto, under the supervision of Professor Peter Scherk. My thanks are due to him for his continued help in the preparation of this paper. I would also like to thank Professor 0 . Haupt for bringing to my attention the theory of linearly connected sets.

1. Curves in $P^{2}$. The theory of hypersurfaces is based on that of curves in the real projective plane. In this chapter, we give a precise definition of the curves of order two and introduce tangents.

1.1 Introduction. Let $P^{n}$ be a real projective space of $n$ dimensions; $n \geqslant 1$. We define a topology of $P^{n}$ in the usual manner. Thus, $P^{n}$ is compact and connected.

Received by the editors April 7, 1975.

AMS (MOS) subject classifications (1970). Primary 53C75; Secondary 53C75.

Key words and phrases. Index, degree of degeneracy, tangent hyperplanes, boundaries of linearly connected sets, ruled quadrics. 
Let $P^{k}, Q^{k}, \ldots$ denote $k$-flats in $P^{n} ;-1 \leqslant k \leqslant n-1$. The $(n-1)$ - and 0 -flats in $P^{n}$ are the hyperplanes and the points of $P^{n}$ respectively. The unique $(-1)$-flat is denoted by $\varnothing$.

For a collection of flats $P^{k}, P^{m}, \ldots, P^{r},\left[P^{k}, P^{m}, \ldots, P^{r}\right]$ denotes the flat in $P^{n}$ spanned by them.

1.2. Plane curves.

1.2.1. A parameter curve $C$ is a continuous map from $P^{1}=\left\{t, t^{\prime}, \ldots\right\}$ into $P^{2}$. A line $T$ is the tangent of a parameter curve $C$ at a point $t \in P^{1}$ if $T=\lim _{t^{\prime} \rightarrow t ; t^{\prime} \neq t}\left[C(t), C\left(t^{\prime}\right)\right]$.

1.2.2. The parameter curve $C$ is differentiable if the tangent of $C$ at every $t \in P^{1}$ exists. $C$ is degenerate if $C$ is injective and $C\left(P^{1}\right)$ is a line. Finally, $C$ is totally degenerate if $C\left(P^{1}\right)$ is a point.

If $C$ is degenerate, then $C\left(P^{1}\right)$ is the tangent of $C$ at $t$ for each $t \in P^{1}$.

1.2.3. A (plane) curve $\Gamma$ is the union of a finite collection of sets $C_{\sigma}\left(P^{1}\right)$ where the $C_{\sigma}$ 's are parameter curves.

A line $T$ is a tangent of $\Gamma$ at $p$ if $T$ is the tangent of some $C_{\sigma}$ at $t$ where $p=C_{\sigma}(t) \in C_{\sigma}\left(P^{1}\right) \subset \Gamma$.

\subsection{Order.}

1.3.1. A differentiable parameter curye $C$ is of order 2 if 2 is the maximum of the number of points of $P^{1}$ mapped into collinear points by $C$ and if a line meets $C\left(P^{1}\right)$ at exactly one point if and only if it the tangent of $C$ at that point.

As a parameter curve is a closed curve, this implies that a differentiable parameter curve $C$ of order 2 is injective and there is a unique tangent at each point of $C\left(P^{1}\right)$.

1.3.2. Let $\Gamma$ be a plane curve. Then $\Gamma$ is of order 1 if $\Gamma=C\left(P^{1}\right)$ where $C$ is a degenerate parameter curve. $\Gamma$ is a nondegenerate curve of order 2 if $\Gamma=C\left(P^{1}\right)$ where $C$ is a differentiable parameter curve of order 2 . Finally, $\Gamma$ is a degenerate curve of order 2 if either $\Gamma=C\left(P^{1}\right)$ where $C$ is a totally degenerate parameter curve or $\Gamma=C_{1}\left(P^{1}\right) \cup C_{2}\left(P^{1}\right)$ where $C_{1}$ and $C_{2}$ are distinct degenerate parameter curves.

We refer to the plane curves in 1.3.2 as the curves of order $\leqslant 2$. We shall denote a nondegenerate plane curve of order 2 by $S^{1}$. We quote without proof:

1.3.3. LEMmA. Let $S^{1} \subset P^{2}$ be a curve of order 2 .

(1) $A$ line $T$ is the tangent of $S^{1}$ at $p$ if and only if $T \cap S^{1}=\{p\}$.

(2) There is a line $L \subset P^{2}$ such that $L \cap S^{1}=\varnothing$.

2. Hypersurfaces of order two. We shall study the (differentiable) hypersurfaces $S^{n-1}$ of order two by constructing their tangent hyperplanes and introducing two invariants: the index and the degree of degeneracy of an $S^{n-1}$. 


\subsection{Introduction.}

2.1.1. A hypersurface of order two in $P^{2}$ is an $S^{1}$. We wish to define hypersurfaces $S^{n-1}$ of order two in $P^{n} ; n \geqslant 3$.

2.1.2. A set $S \subset P^{n}(n \geqslant 3)$ is a hypersurface in $P^{n}$ if it is compact and if every point $p \in S$ has a neighbourhood in $S$ which is the continuous image of the union of a finite number of open $(n-1)$-balls such that outside an $(n-2)$ dimensional subset of that union, the mapping is locally homeomorphic.

In view of 2.1.1, we may assume that $S^{m-1}$, a hypersurface of order 2 in $P^{m}$, is already defined; $2 \leqslant m \leqslant n-1$.

2.1.3. Let $n \geqslant 3$. Let $M$ be a set in $P^{n} ; P^{k} \subset P^{n}, 2 \leqslant k \leqslant n-1$. The $k$-section $P^{k} \cap M$ is

(1) nondegenerate if $P^{k} \cap M$ is an $S^{k-1}$;

(2) degenerate if $P^{k} \cap M$ is either an $m$-flat or a pair of distinct $(k-1)$ flats; $-1 \leqslant m \leqslant k-1$.

2.1.4. A hypersurface $S^{n-1} \subset P^{n}(n \geqslant 3)$ is of order 2 if every intersection of $S^{n-1}$ with a hyperplane is either degenerate or nondegenerate and there is a hyperplane $P_{0}^{n-1}$ such that $P_{0}^{n-1} \cap S^{n-1}$ is an $S^{n-2}$.

Henceforth, $S^{n-1}$ will be a hypersurface of order $2 ; n \geqslant 3$.

2.1.5. Lemma. Let $P^{k} \subset P^{n}, 1 \leqslant k \leqslant n-2$. Then $P^{k} \cap S^{n-1}$ is a flat or a pair of $(k-1)$-flats or an $S^{k-1}$.

Proof. Let $P^{n-1}$ be a hyperplane through $P^{k}$. By 2.1.4, $P^{n-1} \cap S^{n-1}$ is either degenerate or nondegenerate.

If $P^{n-1} \cap S^{n-1}$ is degenerate, then our assertion is trivial.

Suppose $P^{n-1} \cap S^{n-1}$ is an $S^{n-2}$. Let $n=3$. Then $P^{2} \cap S^{2}$ is an $S^{1}$ and the lemma follows from $\S 1$. Assume that the lemma is true for $P^{k} \subset P^{m}$; $3 \leqslant m \leqslant n-1$.

Since $P^{n-1} \cap S^{n-1}$ is an $S^{n-2}$, we have $P^{k} \cap S^{n-1}=P^{k} \cap S^{n-2}$. If $k=n-2$, then $P^{n-2} \cap S^{n-2}$ is either degenerate or nondegenerate by 2.1.4. If $k<n-2$, the lemma follows by the induction hypothesis.

COROLlary. (1) The plane section $P^{2} \cap S^{n-1}$ is either a flat or a curve of order 2.

(2) Any line, not lying in $S^{n-1}$, meets $S^{n-1}$ at most twice.

2.1.6. Let $H^{n-1}$ be a hypersurface in $P^{n}$ such that $P^{n-1} \cap H^{n-1}$ is either a flat or a pair of $(n-2)$-flats for all $P^{n-1} \subset P^{n}$. It is immediate that $H^{n-1}$ is either a hyperplane or a pair of distinct hyperplanes.

2.2. Differentiability. Let $p \in P^{k} \cap S^{n-1}, 2 \leqslant k \leqslant n-1$. If there is a $P^{2}$ such that $p \in P^{2} \subset P^{k}, P^{2} \not \subset S^{n-1}$ and $P^{2} \cap S^{n-1} \neq\{p\}$, then $P^{k} \cap$ $S^{n-1}$ is proper at $p$. 
If $P^{2} \cap S^{n-1}$ is proper at $p$, then there is a tangent of $P^{2} \cap S^{n-1}$ at $p$ by 2.1.5, Corollary and 1.2.3.

2.2.1. A line is a tangent of $S^{n-1}$ at $p$ if it is a tangent of $P^{2} \cap S^{n-1}$ at $p$ for some $P^{2}$ through $p$.

2.2.2. LEMMA. $S^{n-1}$ has a tangent at $p$ for all $p$.

Proof. Let $p$ and $q$ be distinct points of $S^{n-1}$. By 2.1.2, there is a point $r \in P^{n} \backslash S^{n-1}$. If $[p, q, r] \subset P^{2}$, then $P^{2} \cap S^{n-1}$ is proper at $p$.

2.2.3. A point $p \in S^{n-1}$ is differentiable if there is a hyperplane $\pi$ containing all the tangents of $S^{n-1}$ at $p$. Otherwise, $p$ is a singular point of $S^{n-1}$.

2.2.4. LеммA. Let $p \neq q$ in $S^{n-1}$. Then $[p, q]$ is a tangent of $S^{n-1}$ at $p$ if and only if $[p, q] \subset S^{n-1}$.

Proof. Let $[p, q]$ be a tangent of $S^{n-1}$ at $p$. Then $[p, q]$ is a tangent of $P^{2} \cap S^{n-1}$ at $p$ for some $P^{2}$ through $[p, q]$. If $P^{2} \cap S^{n-1}$ is a curve of order 1 , then $[p, q]=P^{2} \cap S^{n-1}$. If $P^{2} \cap S^{n-1}$ is a curve of order 2 , then $P^{2} \cap S^{n-1}$ is degenerate by 1.3.3. Thus, $P^{2} \cap S^{n-1}$ is a pair of lines, one of which is $[p, q]$.

Conversely, let $[p, q] \subset S^{n-1}$. Choose a point $r \in P^{n} \backslash S^{n-1}$. Then $[p, q]$ is a tangent of $[p, q, r] \cap S^{n-1}$ at $p$.

2.2.5. TheOREM. Let $p \in S^{n-1}$ be differentiable. Then every line through $p$ in $\pi$ is a tangent of $S^{n-1}$ at $p ; c f .2 .2 .3$. In particular, the tangent hyperplane $\pi=\pi(p)$ of $S^{n-1}$ at $p$ is unique.

Proof. Let $L$ be a line; $p \in L \subset \pi$. Choose a point $q \in S^{n-1} \backslash \pi$. By 2.2.4, $[p, q] \not \subset S^{n-1}$. Then $[L, q] \cap S^{n-1}$ is proper at $p$ with a tangent $T$ at p. As $T \subset \pi$, we have $T=\pi \cap[L, q]=L$.

2.2.6. $S^{n-1}$ is differentiable if each point contained in any $S^{1} \subset S^{n-1}$ is differentiable.

We shall prove that the hypersurfaces of order 2 are in fact all differentiable; cf. 2.2.9.

2.2.7. Lemma. Let $\{p, q\} \subset S^{n-1} ;[p, q] \not \subset S^{n-1}$. Let $T$ be a line such that $T \cap S^{n-1}=\{p\}$. Then $T$ is a tangent of $S^{n-1}$ at $p$.

Proof. Let $P^{2}=[T, q]$. By $2.1 .5, P^{2} \cap S^{n-1}$ is a flat or a pair of lines or an $S^{1}$. Since $T \cap S^{n-1}=\{p\}$, the first two instances imply that $[p, q]$ $\subset S^{n-1}$. Thus, $P^{2} \cap S^{n-1}$ is an $S^{1}$ and by 1.3.3, $T$ is a tangent of $S^{1}$ at $p$.

2.2.8. Lemma. Let $\{p, q\} \subset S^{n-1} ;[p, q] \not \subset S^{n-1}$. Then $\tau(p)=\{r \in$ $P^{n} \mid r$ lies on a tangent of $S^{n-1}$ at $\left.p\right\}$ is a flat. 
Proof. By 2.2.2, $\tau(p) \neq \varnothing$. Let $r_{1} \neq r_{2}$ be points in $\tau(p)$. We may assume that $p \notin\left[r_{1}, r_{2}\right]$. Thus, $P^{2}=\left[r_{1}, r_{2}, p\right]$ is a plane and $T_{i}=\left[p, r_{i}\right]$ are distinct tangents of $S^{n-1}$ at $p ; i=1,2$.

If $T_{1} \cup T_{2} \subset S^{n-1}$, then either $P^{2} \subset S^{n-1}$ or $P^{2} \cap S^{n-1}=T_{1} \cup T_{2}$. If say $T_{1} \subset S^{n-1}$ and $T_{2} \not \subset S^{n-1}$, then either $P^{2} \cap S^{n-1}=T_{1}$ or $P^{2} \cap S^{n-1}$ is a pair of lines. But $T_{2} \not \subset S^{n-1}$ implies that $T_{2} \cap S^{n-1}=\{p\}$ by 2.2.4. Thus if $P^{2} \cap S^{n-1}$ is a pair of lines, then both lines pass through $p$.

If $T_{i} \cap S^{n-1}=\{p\} ; i=1,2$, then $T_{1} \neq T_{2}$ implies that $P^{2} \cap S^{n-1}$ is not an $S^{1}$ by 1.3.3. Hence $P^{2} \cap S^{n-1}$ is the point $p$ or a line or a pair of lines, all through $p$.

Let $T$ be a line; $p \in T \subset P^{2}$. By the preceding, either $T \subset S^{n-1}$ or $T \cap$ $S^{n-1}=\{p\}$. Then $T \subset \tau(p)$ by 2.2 .4 and 2.2 .7 respectively. Hence, $\left[r_{1}, r_{2}\right] \subset$ $P^{2} \subset \tau(p)$ and $\tau(p)$ is a flat.

\subsubsection{THEOREM. $S^{n-1}$ is differentiable; $n \geqslant 3$.}

Proof. Let $P^{2}$ be a plane such that $P^{2} \cap S^{n-1}$ is an $S^{1}$. Let $p \in S^{1}$. Then $[p, q] \not \subset S^{n-1}$ for each $q \in S^{1} \backslash\{p\}$ and thus, $q \notin \tau(p)$ by 2.2.4. Hence, $\operatorname{dim} \tau(p) \leqslant n-1$ and there is a hyperplane $\pi$ containing $\tau(p)$.

2.2.10. THEOREM. A point $v \in S^{n-1}$ is singular if and only if $[v, p] \subset$ $S^{n-1}$ for all $p \in S^{n-1}$.

Proof. From 2.1.4, it is immediate that there is a plane $P_{0}^{2}$ such that $P_{0}^{2} \cap S^{n-1}$ is an $S^{1}$. By 1.3.3, there is a line $L \subset P_{0}^{2}$ such that $L \cap S^{1}=$ $L \cap S^{n-1}=\varnothing$.

Let $v \in S^{n-1}$ be singular. By 2.2.6 and 2.2.9, $v \notin S^{1}$. Then by 2.1.5 Corollary, $[L, v] \cap S^{n-1}=\{v\}$.

Let $p \in S^{n-1} \backslash\{v\}$. Then clearly, $P^{3}=[L, v, p]$ is a 3-flat. Let $P^{2} \subset P^{3}$ be a plane through $[v, p]$. By 2.2.6 and 2.2.9, $P^{2} \cap S^{n-1}$ is either a line or a pair of lines. As the line $P^{2} \cap[L, v]$ meets $S^{n-1}$ only at $v$, every line in $P^{2} \cap S^{n-1}$ passes through $v$. Thus, $[v, p] \subset S^{n-1}$.

Since $S^{n-1}$ is not contained in any hyperplane, the converse follows by 2.2.4 and 2.2.3.

COROLlary. The set $V$ of all the singular points of $S^{n-1}$ is a flat; moreover, $V \subset \pi(p)$ for all $p \in S^{n-1} \backslash V$.

2.2.11. $S^{n-1}$ is $d$-(times) degenerate if $\operatorname{dim} V=d-1$. Obviously, $0 \leqslant$ $d \leqslant n-2$. For brevity, $S^{n-1}$ is [non] degenerate if [d=0] $d>0$.

2.3. The index of $S^{n-1}$.

2.3.1. $S^{n-1}$ has the index $i$ if $i=$ ind $S^{n-1}$ is the maximum dimension of any flat of $P^{n}$ contained in $S^{n-1}$. 
2.3.2. REMARK. $\operatorname{dim} V<$ ind $S^{n-1} \leqslant n-2$.

Let $M$ be a set in $P^{n}$. Then [M] is the flat spanned by the points of $M$.

2.3.3. Lemma. Let ind $S^{n-1}=i$. Then every point of $S^{n-1}$ lies in an i-flat contained in $S^{n-1}$.

Proof. Since ind $S^{n-1}=i$, there is an $i$-flat $P^{i} \subset S^{n-1}$.

Let $v \in V$. By 2.2.10, $\left[P^{i}, v\right]$ is a flat in $S^{n-1}$ and thus, $i \leqslant \operatorname{dim}\left[P^{i}, v\right] \leqslant$ ind $S^{n-1}=i$. Hence, $\left[P^{i}, v\right]=P^{i}$ and $V \subset P^{i}$.

Let $p \in S^{n-1} \backslash P^{i}$. Then $p$ is differentiable and $p \notin \pi(p) \cap P^{i}$. By 2.2.4, $\left[p, \pi(p) \cap P^{i}\right] \subset S^{n-1}$. Clearly, $\operatorname{dim}\left(\pi(p) \cap P^{i}\right)=i-1$ and $\operatorname{dim}\left[p, \pi(p) \cap P^{i}\right]=i$.

Corollary 1. Let ind $S^{n-1}=i ; p \in S^{n-1} \backslash V$. Then

(1) $P^{i} \subset \pi(p) \cap S^{n-1}$ if and only if $p \in P^{i} \subset S^{n-1}$;

(2) $V=\bigcap_{P i \subset S^{n-1}} P^{i}$.

Proof. The proof of 2.3.3 implies (1) and $V \subset \bigcap_{P^{i} \subset S^{n-1}} P^{i}=W$.

Let $q \in W$ and let $p \in S^{n-1}$. By 2.3.3,p lies in an $i$-flat $P_{0}^{i} \subset S^{n-1}$. Thus, $[p, q] \subset P_{0}^{i} \subset S^{n-1}$ and by 2.2.10,q $\in$.

Corollary 2. Let $\pi(p) \cap S^{n-1}=S^{n-2}$ for some $p \in S^{n-1} \backslash V$. Then ind $S^{n-1} \leqslant n-3$.

Proof. By 2.1.4, there are no $(n-2)$-flats in $S^{n-2}$. Thus $p$ does not lie in an $(n-2)$-flat. By 2.3.3, ind $S^{n-1} \leqslant n-3$.

2.3.4. Lemma. Let $\{p, q\} \subset S^{n-1} ;[p, q] \not \subset S^{n-1}$. Then $\operatorname{dim}\left[\pi(p) \cap S^{n-1}\right]=\operatorname{dim}\left[\pi(q) \cap S^{n-1}\right]$.

Proof. Let $k=\operatorname{dim}\left[\pi(p) \cap S^{n-1}\right]$. By the symmetry in $p$ and $q$, it is sufficient to prove that $k \leqslant \operatorname{dim}\left[\pi(q) \cap S^{n-1}\right]$. Thus, we may assume $k>0$.

Choose points $p_{\sigma} \in \pi(p) \cap S^{n-1}$ such that $\left[\pi(p) \cap S^{n-1}\right]=\left[p, p_{1}\right.$, $\left.\ldots, p_{k}\right]$. By $2.2 .4, L_{\sigma}=\left[p, p_{\sigma}\right] \subset S^{n-1}$ and $q \notin \pi(p)$. Thus, $\left[L_{\sigma}, q\right] \cap$ $S^{n-1}$ contains the line $\left[q, q_{\sigma}\right]$ for some $q_{\sigma} \in L_{\sigma}$. Then $q_{\sigma} \in \pi(q)$ and $\left[q, q_{1}, \ldots, q_{k}\right] \subset\left[\pi(q) \cap S^{n-1}\right]$. Since $[p, q] \not \subset S^{n-1}$, we have $q \neq q_{\sigma} \neq p$ for $\sigma=1, \ldots, k$. Thus, $\left[\pi(p) \cap S^{n-1}\right]=\left[L_{1}, L_{2}, \ldots, L_{k}\right]=\left[p, q_{1}\right.$, $\left.\ldots, q_{k}\right]$ and $P^{k-1}=\left[q_{1}, \ldots, q_{k}\right]$ is a $(k-1)$-flat. Obviously, $q \notin P^{k-1}$ and thus the $k$-flat $\left[q, p^{k-1}\right] \subset \pi(q) \cap S^{n-1}$. Hence, $\operatorname{dim}\left[\pi(q) \cap S^{n-1}\right] \geqslant k$.

2.3.5. REMARK. Let $p \in S^{n-1} \backslash V$. Then

(1) $\pi(p) \cap S^{n-1}$ is a $k$-flat if and only if $\operatorname{dim}\left[\pi(p) \cap S^{n-1}\right]=k, 0 \leqslant$ $k \leqslant n-2$;

(2) $\pi(p) \cap S^{n-1}$ is an $S^{n-2}$ if and only if $\operatorname{dim}\left[\pi(p) \cap S^{n-1}\right]=n-1$ and ind $S^{n-1}<n-2$.

2.3.6. TheOREM. Let ind $S^{n-1}=i ; 0 \leqslant i \leqslant n-2$. Then we have 
precisely one of the following three cases:

(1) $\pi(p) \cap S^{n-1}$ is an i-flat for all $p \in S^{n-1} \backslash V$;

(2) $\pi(p) \cap S^{n-1}$ is a pair of distinct $(n-2)$-flats for all $p \in S^{n-1} \backslash V$, $i=n-2$;

(3) $\pi(p) \cap S^{n-1}$ is an $S^{n-2}$ for all $p \in S^{n-1} \backslash V, 0<i<n-2$.

Proof. Let $p \in S^{n-1} \backslash V$. By 2.3.3, there is a $P^{i}$ through $p$ in $S^{n-1}$. By 2.2.4, $P^{i} \subset \pi(p)$ and thus, $k(p)=\operatorname{dim}\left[\pi(p) \cap S^{n-1}\right] \geqslant i$. By 2.3.5, either $k(p)=i$ or $k(p)=n-1$. It remains to be shown that $k(p)$ is independent of $p$.

Let $\left\{p_{1}, p_{2}\right\} \subset S^{n-1} \backslash V ; p_{1} \neq p_{2}$. If $\left[p_{1}, p_{2}\right] \not \subset S^{n-1}$, then 2.3 .4 implies that $k\left(p_{1}\right)=k\left(p_{2}\right)$. Let $\left[p_{1}, p_{2}\right] \subset S^{n-1}$. Since $S^{n-1} \backslash V \not \subset \pi\left(p_{1}\right) \cup \pi\left(p_{2}\right)$, there is a $p_{3} \in S^{n-1} \backslash V$ such that $p_{3} \notin \pi\left(p_{1}\right) \cup \pi\left(p_{2}\right)$. Therefore, $k\left(p_{1}\right)=$ $k\left(p_{3}\right)=k\left(p_{2}\right)$.

2.4. Nondegenerate $S^{n-1}$. In this and the following section, we examine the properties of the nondegenerate and the degenerate $S^{n-1}$ and we consider a relationship between them.

2.4.1. Lemma. Let $S^{n-1}$ be nondegenerate; ind $S^{n-1}=i$. Then either $i=0$ or $\pi(p)=\left[\pi(p) \cap S^{n-1}\right]$ for $p \in S^{n-1}$.

Proof. Let $\left\{p_{0}, p_{1}\right\} \subset S^{n-1} ;\left[p_{0}, p_{1}\right] \not \subset S^{n-1}$. By 2.3.3, there is a $P_{\sigma}^{i}, p_{\sigma} \in P_{\sigma}^{i} \subset S^{n-1} ; \sigma=0,1$. Clearly, $P_{0}^{i} \neq P_{1}^{i}$.

Suppose a $q \in P_{0}^{i} \cap P_{1}^{i}$. Then $P_{0}^{i} \cup P_{1}^{i} \subset \pi(q) \cap S^{n-1}$ and by 2.3.6, $\left[\pi(q) \cap S^{n-1}\right]=\pi(q)$. Assume that $\left[\pi(p) \cap S^{n-1}\right] \neq \pi(p)$ for some and hence for all $p \in S^{n-1}$ (2.3.6). Then by the preceding, $P_{0}^{i} \cap P_{1}^{i}=\varnothing$. By 2.3.6, $\pi\left(p_{0}\right) \cap S^{n-1}=P_{0}^{i}$ and thus, $\pi\left(p_{0}\right) \cap P_{1}^{i}=\varnothing$. Hence, $P_{1}^{i}=\left\{p_{1}\right\}$ and $i=0$.

2.4.2. LeMMA. Let $\left\{p_{0}, p_{1}\right\} \subset S^{n-1} \backslash V ; P^{1}=\left[p_{0}, p_{1}\right] \subset S^{n-1}$. Then $\pi\left(p_{0}\right) \cap \pi\left(p_{1}\right) \subseteq \pi(p)$ for all $p \in P^{1} \backslash V$.

Proof. Let $u \in\left(\pi\left(p_{0}\right) \cap \pi\left(p_{1}\right)\right) \backslash P^{1}$.

If $u \in S^{n-1}$, then $\left[P^{1}, u\right] \cap S^{n-1}$ contains the lines $P^{1},\left[p_{0}, u\right]$ and $\left[p_{1}, u\right]$ by 2.2.4. Thus, $\left[P^{1}, u\right] \subset S^{n-1}$ by 2.1 .5 .

If $u \notin S^{n-1}$, then $\left[p_{\sigma}, u\right] \cap S^{n-1}=\left\{p_{\sigma}\right\}$ by 2.2.4. Hence, 2.1 .5 implies that $\left[P^{1}, u\right] \cap S^{n-1}=P^{1}$. Thus, $[p, u] \cap S^{n-1}=\{p\}$ for all $p \in P^{1}$ and the lemma follows by 2.2 .7 .

2.4.3. Lemma. Let $S^{n-1}$ be nondegenerate. Let $P^{k}=\left[p_{0}, \ldots, p_{k}\right] \subset$ $S^{n-1}$. Then $M=\bigcap_{\sigma=0}^{k} \pi\left(p_{\sigma}\right)$ is an $(n-k-1)$-flat.

Proof. Our statement is trivial for $k=0$.

Assume that the lemma is true for any $P^{k-1} \subset S^{n-1}$. Since $\left[p_{0}, \ldots\right.$, $\left.p_{k-1}\right] \subset S^{n-1}$, we have that $P_{1}^{n-k}=\bigcap_{j=0}^{k-1} \pi\left(p_{j}\right)$ is an $(n-k)$-flat. Then $M=\pi\left(p_{k}\right) \cap P_{1}^{n-k}$ and $n-k-1 \leqslant \operatorname{dim} M \leqslant n-k$. 
Let $q \in S^{n-1} \backslash \bigcup_{j=0}^{k} \pi\left(p_{j}\right)$. Then $\left[p_{k}, p_{\sigma}, q\right]$ is not in $S^{n-1}$ and there is a line $L_{\sigma} \subset\left[p_{k}, p_{\sigma}, q\right] \cap S^{n-1}$ such that $L_{\sigma}=\left[q, q_{\sigma}\right]$ for some $q_{\sigma} \in\left[p_{k}, p_{\sigma}\right]$; $\sigma=0,1, \ldots, k-1$. By 2.2.4, $p_{k} \neq q_{\sigma} \neq p_{\sigma}$ and $q \in \pi\left(q_{\sigma}\right)$.

By our construction, $P^{k}=\left[p_{0}, p_{1}, \ldots, p_{k}\right]=\left[q_{0}, q_{1}, \ldots, q_{k-1}, p_{k}\right]$. Thus, $\left[q_{0}, \ldots, q_{k-1}\right] \subset S^{n-1}$ is a $(k-1)$-flat and by the induction hypothesis, $\bigcap_{\sigma=0}^{k-1} \pi\left(q_{\sigma}\right)=P_{2}^{n-k}$ is an $(n-k)$-flat.

By 2.4.2, $q_{\sigma} \in\left[p_{k}, p_{\sigma}\right] \subset S^{n-1}$ implies that $\pi\left(p_{k}\right) \cap \pi\left(p_{\sigma}\right) \subseteq \pi\left(q_{\sigma}\right) ;$ $\sigma=0,1, \ldots, k-1$. Thus, $M=\pi\left(p_{k}\right) \cap P_{1}^{n-k} \subseteq P_{2}^{n-k}$ and $q \in P_{2}^{n-k} \backslash M$.

Since $P^{k} \subset M$ in 2.4.3, we have

2.4.4. THEOREM. Let $S^{n-1}$ be nondegenerate. Then ind $S^{n-1} \leqslant 1 / 2(n-1)$.

CoROllaRY 1. If $n=3$, then ind $S^{2}=0$ or 1 and $\pi(p) \cap S^{2}$ is the point $p$ or a pair of distinct lines through $p$ respectively. $S^{n-2}$.

COROLlaRY 2. If $n \geqslant 4$ and ind $S^{n-1}>0$, then $\pi(p) \cap S^{n-1}$ is an

It should be noted that our results concerning the surfaces of order 2 in $P^{3}$ coincide with the theory in [2]. Of particular importance is the following result of Marchaud. While his proof is incomplete, it is easy to verify.

2.4.5. ThEorem (MARChaud [2]). A nondegenerate $S^{2}$ with the index 1 is a quadric.

2.5. Degenerate $S^{n-1}$. Let $S^{n-1}$ be $d$-degenerate; ind $S^{n-1}=i$. Thus, $\operatorname{dim} V=d-1$ and $0 \leqslant d \leqslant i \leqslant n-2$. Put $m=n-d$ and let $P_{v}^{m}$ be an $m$-flat such that $P_{v}^{m} \cap V=\varnothing$. Then $P^{n}=\left[V, P_{v}^{m}\right]$.

2.5.1. LEMMA. $S^{n-1}=\bigcup[V, p], p \in P_{v}^{m} \cap S^{n-1}$.

Proof. Let $q \in S^{n-1} \backslash V$. Then $[V, q] \cap P_{v}^{m}$ is a point $p$ and $[V, q]=$ $[V, p] \subset S^{n-1}$.

2.5.3. Lemma. $P_{v}^{m} \cap S^{n-1}$ is a nondegenerate $S^{m-1}$.

Proof. Let $p \in P_{v}^{m} \cap S^{n-1}$ (2.5.1). By 2.2.10,p£V implies that there is a $p^{\prime} \in S^{n-1}$ such that $\left[p, p^{\prime}\right] \not \subset S^{n-1}$. Hence $p^{\prime} \notin V$. By 2.5.1, $p^{\prime} \in[v, q]$ where $v \in V$ and $q \in P_{v}^{m} \cap S^{n-1}$. Thus, $\left[v, p, p^{\prime}\right]$ is a plane and by 2.1 .5 and 2.2.10, $\left[v, p, p^{\prime}\right] \cap S^{n-1}=[v, p] \cup\left[v, q, p^{\prime}\right]$. Thus, $[p, q] \cap P_{v}^{m} \cap S^{n-1}=$ $\{p, q\}$ and $P_{v}^{m} \cap S^{n-1}$ is not a flat.

Let $P^{k} \subset P_{v}^{m} \cap S^{n-1}$. Then 2.2 .10 and $P^{k} \cap V=\varnothing$ imply that the $(k+d)$-flat $\left[V, P^{k}\right]$ lies in $S^{n-1}$. Thus, $k+d \leqslant i \leqslant n-2$ and $k \leqslant i-d \leqslant$ $n-d-2=m-2$.

By 2.1.5, $P_{v}^{m} \cap S^{n-1}$ is an $S^{m-1}$. By 2.2.10 and the preceding, $S^{m-1}$ is nondegenerate. 
2.5.3. LEMMA, ind $S^{m-1}=i-d$.

Proof. Since ind $S^{n-1}=i$, there is a $P^{i} \subset S^{n-1}$. Then $V \subset P^{i}$ and $P^{n}=\left[P^{i}, P_{v}^{m}\right]$. Thus, $P^{i} \cap P_{v}^{m}$ is an $(i-d)$-flat contained in $S^{m-1}$.

From the proof of 2.5 .2 , ind $S^{m-1} \leqslant i-d$.

In summary, we have

2.5.4. THEOREM. Let $S^{n-1}$ be d-degenerate; ind $S^{n-1}=i$. Let $P^{n-d} \cap$ $V=\varnothing$. Then $P^{n-d} \cap S^{n-1}$ is a nondegenerate $S^{n-d-1}$ with the index $i-d$ and $S^{n-1}=\bigcup_{p \in S^{n-d-1}}[V, p]$.

This theorem reduces the study of a degenerate $S^{n-1}$ to that of a suitable nondegenerate $S^{n-d-1}$. But in 2.4, we have already classified the latter according to the index.

2.5.5. THEOREM. Let $S^{n-1}$ be d-degenerate; ind $S^{n-1}=i$. Let $p \in$ $S^{n-1} \backslash V$ and let $P^{n-d} \cap V=\varnothing ; p \in P^{n-d}$. Then there are exactly the following three cases:

(1) $\pi(p) \cap S^{n-d-1}=\{p\}$. Then $\pi(p) \cap S^{n-1}=[V, p]$ and $i=d$.

(2) $\pi(p) \cap S^{n-d-1}=P_{1}^{1} \cup P_{2}^{1}, P_{1}^{1} \neq P_{2}^{1} ; n-d=3$. Then $\pi(p) \cap S^{n-1}$ $=P_{1}^{n-2} \cup P_{2}^{n-2}$ where $P_{\sigma}^{n-2}=\left[P_{\sigma}^{1}, V\right] ; \sigma=1,2$.

(3) $\pi(p) \cap S^{n-d-1}=S^{n-d-2} ; n-d \geqslant 4$. Then $\pi(p) \cap S^{n-1}$ is an $S^{n-2}$ and $d<i \leqslant 1 / 2(n+d-1)$.

Proof. Let $\pi^{\prime}(p)$ be the tangent hyperplane of $S^{n-d-1}$ at $p$. Then $\pi^{\prime}(p)=\pi(p) \cap P^{n-d}$ and $\pi^{\prime}(p) \cap S^{n-d-1}=\pi(p) \cap S^{n-d-1}$. As $S^{n-d-1}$ is nondegenerate, we obtain the intersections $\pi^{\prime}(p) \cap S^{n-1}$ by 2.4.1 and 2.4.4.

(1) If $\pi(p) \cap S^{n-d-1}=\{p\}$, then ind $S^{n-d-1}=i-d=0$. Since $\operatorname{dim} V=d-1,[V, p] \subset \pi(p) \cap S^{n-1}$ is a $d$-flat. Clearly, ind $S^{n-1}=d \mathrm{im}$ plies that $\pi(p) \cap S^{n-1}=[V, p]$.

(2) If $\pi(p) \cap S^{n-d-1}=P_{1}^{1} \cup P_{2}^{1}$, then ind $S^{n-d-1}=i-d=1$ and $n-d=3$. Thus, $i=n-2$ and the result follows by 2.3.6.

(3) If $\pi(p) \cap S^{n-d-1}=S^{n-d-2}$, then $S^{n-d-2} \subset \pi(p) \cap S^{n-1}, n-d$ $\geqslant 4$ and $0<i-d \leqslant 1 / 2(n-d-1)$ by 2.4.4. Obviously, $\pi(p) \cap S^{n-1}$ is an $S^{n-2}$.

2.6. Decomposition. To facilitate the study of nondegenerate $S^{n-1}$,s, we shall decompose them, whenever possible, into nondegenerate hypersurfaces of smaller dimension.

Let $S^{n-1}$ be nondegenerate; ind $S^{n-1}=i \geqslant 1$ and $n \geqslant 4$.

2.6.1. LemmA. For any $p \in S^{n-1}, \pi(p) \cap S^{n-1}$ is a 1-degenerate $S^{n-2}$ with ind $S^{n-2}=i$ and the singular point $p$.

Proof. By 2.5.5, $\pi(p) \cap S^{n-1}$ is an $S^{n-2}$. Since ind $S^{n-1}=i$, there is 
a $P^{i} \subset \pi(p) \cap S^{n-1}$ and thus, ind $S^{n-2}=i$.

Let $q \in S^{n-2}$. By 2.2.4, $[p, q] \subset \pi(p) \cap S^{n-1}=S^{n-2}$ and by 2.2.10, $p$ is a singular point of $S^{n-2}$.

If $p \neq q$, then $\pi(p) \neq \pi(q)$ by 2.4 .3 . Hence by 2.4 .1 ,

$$
\left[\pi(q) \cap S^{n-1}\right]=\pi(q) \neq \pi(p)=\left[\pi(p) \cap S^{n-1}\right]=\left[S^{n-2}\right] .
$$

Thus, there is a point $u \in S^{n-2}$ such that $[u, q] \not \subset S^{n-1}$. In particular, $[u, q]$ $\not \subset S^{n-2}$ and $S^{n-2}$ is differentiable at $q$ by 2.2 .10 . Hence, $p$ is the only singular point of $S^{n-2}$.

2.6.2. Lemma. Let $\{p, q\} \subset S^{n-1} ;[p, q] \not \subset S^{n-1}$. Then $\pi(p) \cap \pi(q) \cap$ $S^{n-1}$ is a nondegenerate $S^{n-3}$ with ind $S^{n-3}=i-1$.

Proof. Since $[p, q] \not \subset S^{n-1}, P^{n-2}=\pi(p) \cap \pi(q)$ is an $(n-2)$-flat. Note that $P^{n-2} \cap S^{n-1} \subset \pi(p) \cap S^{n-1} \subset S^{n-1}$.

By 2.6.1, $\pi(p) \cap S^{n-1}$ is 1-degenerate with ind $S^{n-1}=i$ and with the singular point $p$. Since $\pi(p)=\left[P^{n-2}, p\right], 2.5 .4$ yields that $P^{n-2} \cap(\pi(p) \cap$ $\left.S^{n-1}\right)=P^{n-2} \cap S^{n-2}$ is a nondegenerate $S^{n-3}$; ind $S^{n-3}=i-1$.

COROllary. $P^{n}=\left[P^{n-2}, p, q\right]$.

2.6.3. THEOREM. There is a sequence of points $p_{0}, q_{0}, \ldots, p_{i}, q_{i}$ in $S^{n-1}$ such that, for $0 \leqslant k \leqslant i$,

(1) $\bigcap_{\sigma=0}^{k-1}\left(\pi\left(p_{\sigma}\right) \cap \pi\left(q_{\sigma}\right)\right)=R^{n-2 k}$ is an $(n-2 k)$-flat,

(2) $\left\{p_{k}, q_{k}\right\}=\left[p_{k}, q_{k}\right] \cap\left(R^{n-2 k} \cap S^{n-1}\right)$, and

(3) if $n-2 k>1$, then $R^{n-2 k} \cap S^{n-1}$ is a nondegenerate $S^{n-2 k-1}$; ind $S^{n-2 k-1}=i-k$.

Proof. Let $p_{0} \in S^{n-1}$. Since $S^{n-1}$ is differentiable, there is a $q_{0} \in$ $S^{n-1}$ such that $\left[p_{0}, q_{0}\right] \not \subset S^{n-1}$ by 2.2.10. Then $R^{n-2}=\pi\left(p_{0}\right) \cap \pi\left(p_{0}\right)$ is an $(n-2)$-flat and by $2.6 .2, R^{n-2} \cap S^{n-1}$ is a nondegenerate $S^{n-3}$; ind $S^{n-3}$ $=i-1$. We now choose points $\left\{p_{1}, q_{1}\right\} \subset S^{n-3}$ such that $\left[p_{1}, q_{1}\right] \not \subset S^{n-3}$. The tangent hyperplane of $S^{n-3}$ is the $(n-3)$-flat $\pi(p) \cap R^{n-2}$; thus

is an $(n-4)$-flat.

$$
R^{n-4}=\pi\left(p_{1}\right) \cap \pi\left(q_{1}\right) \cap R^{n-2}=\bigcap_{\sigma=0}^{1}\left(\pi\left(p_{\sigma}\right) \cap \pi\left(q_{0}\right)\right)
$$

If $n \geqslant 6$ and $i \geqslant 2$, we can repeat this construction. By 2.6.2, $R^{n-4} \cap$ $S^{n-3}=R^{n-4} \cap S^{n-1}$ is a nondegenerate $S^{n-5}$; ind $S^{n-5}=i-2$. Obviously, we can choose $\left\{p_{2}, q_{2}\right\} \subset S^{n-5}$ such that $\left[p_{2}, q_{2}\right] \not \subset S^{n-5}$.

Thus, as long as $n-2(k-1) \geqslant 4$ and $i \geqslant k$, we can repeat our construction obtaining a sequence of points $p_{0}, q_{0}, \ldots, p_{k}, q_{k}$ which satisfy the conditions (1)-(3).

By $2.4 .4, n \geqslant 2 i+1$. If $n>2 i+1$, then $i \geqslant k$ implies that $n-2(k-1)$ 
4. Thus, the construction yields $p_{0}, q_{0}, \ldots, p_{i}, q_{i}$.

If $n=2 i+1$, then $i-1 \geqslant k$ implies that $n-2(k-1) \geqslant 5$. Now the construction yields $p_{0}, q_{0}, \ldots, p_{i-1}, q_{i-1}$. In particular, $R^{n-2(i-1)}=R^{3}$ and $R^{3} \cap S^{n-2}$ is a nondegenerate $S^{2}$; ind $S^{2}=1$.

By 2.2.4 Corollary $1, \pi(p) \cap S^{2}$ is a pair of lines through $p$ for $p \in S^{2}$. Since $\left[p_{i-1}, q_{i-1}\right] \not \subset S^{2}$, the line $R^{1}=\pi\left(p_{i-1}\right) \cap \pi\left(q_{i-1}\right) \cap R^{3}$ does not. pass through $p_{i-1}$ or $q_{i-1}$. Thus, $R^{1}$ meets $S^{2}$ at exactly two distinct points, say $p_{i}$ and $q_{i}$. Then $\left[p_{i}, q_{i}\right] \not \subset S^{2}$ and $\left\{p_{i}, q_{i}\right\}=R^{1} \cap S^{2}=\bigcap_{\sigma=0}^{i-1}\left[\left(\pi\left(p_{\sigma}\right) \cap \pi\left(q_{\sigma}\right)\right)\right]$ $\cap S^{n-1}$.

Corollary. $\operatorname{dim}\left[p_{0}, \ldots, p_{i}, q_{0}, \ldots, q_{i}\right]=2 i+1$.

Proof. By 2.6.2 Corollary, we obtain

$$
\begin{aligned}
P^{n} & =\left[R^{n-2}, p_{0}, q_{0}\right]=\left[R^{n-4}, p_{1}, q_{1}, p_{0}, q_{0}\right] \\
& =\left[R^{n-2 i}, p_{i-1}, q_{i-1}, \ldots, p_{0}, q_{0}\right]
\end{aligned}
$$

If $n=2 i+1$, then $R^{n-2 i}=R^{1}=\left[p_{i}, q_{i}\right]$. If $n>2 i+1$, then ind $S^{n-2 i-1}=0$ and $R^{n-2 i}=\left[\pi\left(p_{i}\right) \cap \pi\left(q_{i}\right) \cap R^{n-2 i}, p_{i}, q_{i}\right]$. Since $\left(\pi\left(p_{i}\right) \cap\right.$ $\left.\pi\left(q_{i}\right)\right) \cap\left[p_{i}, q_{i}\right]=\varnothing$, the result follows.

2.6.4. Let $r_{0}, r_{1}, \ldots, r_{k}$ be a sequence of points in $P^{n}$. We shall denote by $\left[r_{0}, \ldots, \hat{r}_{\alpha}, \ldots, r_{k}\right]$, the flat of $P^{n}$ spanned by the points $r_{\sigma} ; \sigma=0,1$, $\ldots, k$ and $\sigma \neq \alpha$.

Let $I=\{0,1, \ldots, i\}$. From 2.6.3, we observe that

(1) $P^{n}=\left[p_{0}, \ldots, p_{i}, R^{s}, q_{0}, \ldots, q_{i}\right]$ where

(2) $R^{s}=\bigcap_{\sigma=0}^{i}\left(\pi\left(p_{\sigma}\right) \cap \pi\left(q_{\sigma}\right)\right)$; $s=n-(2 i+2) \geqslant-1$. Thus for $\alpha \in I$,

(3) $\pi\left(p_{\alpha}\right)=\left[p_{0}, \ldots, p_{i}, R^{s}, q_{0}, \ldots, \hat{q}_{\alpha}, \ldots, q_{i}\right]$ and

(4) $\pi\left(q_{\alpha}\right)=\left[q_{0}, \ldots, q_{i}, R^{s}, p_{0}, \ldots, \hat{p}_{\alpha}, \ldots, p_{i}\right]$.

We put

(5) $\widetilde{P}^{i}=\left[p_{0}, \ldots, p_{i}\right]$,

(6) $\widetilde{Q}^{i}=\left[q_{0}, \ldots, q_{i}\right]$,

(7) $P_{\alpha}^{i}=\left[p_{0}, \ldots, \hat{p}_{\alpha}, \ldots, p_{i}, q_{\alpha}\right], \alpha \in I$, and

(8) $Q_{\alpha}^{i}=\left[q_{0}, \ldots, \hat{q}_{\alpha}, \ldots, q_{i}, p_{\alpha}\right], \alpha \in I$.

Thus, $P^{n}=\left[\widetilde{P}^{i}, R^{s}, \widetilde{Q}^{i}\right]$.

2.6.5. Lemma. The i-flats $\widetilde{P}^{i}, \widetilde{Q}^{i}, P_{\alpha}^{i}$ and $Q_{\alpha}^{i}$ lie in $S^{n-1} ; \alpha \in I$.

Proof. We prove that $\widetilde{P}^{i} \subset S^{n-1}$.

By 2.1.5, $\widetilde{P}^{i} \cap S^{n-1}$ is a flat or a pair of $(i-1)$-flats or an $S^{i-1}$. By 2.6.4(3), $p_{\alpha} \in \pi\left(p_{\beta}\right)$ and thus, $\left[p_{\alpha}, p_{\beta}\right] \subset S^{n-1}$ for $\{\alpha, \beta\} \subset I$ by 2.2.4. Then $\widetilde{P}^{i}=\left[p_{0}, \ldots, p_{i}\right]$ implies that either $\widetilde{P}^{i} \subset S^{n-1}$ or $\widetilde{P}^{i} \cap S^{n-1}$ is an $S^{i-1}$. Suppose $\widetilde{P}^{i} \cap S^{n-1}$ is an $S^{i-1}$. Since $\left[p_{\alpha}, p_{\beta}\right] \subset S^{n-1}$ for $\{\alpha, \beta\} \subset I$, 
$p_{\alpha}$ must be a singular point of $S^{i-1}$ for $\alpha \in I$. Thus, $\widetilde{P}^{i}$ is the singular flat of $S^{i-1}$; a contradiction. Thus $\widetilde{P}^{i} \subset S^{n-1}$.

By similar arguments, the other $i$-flats lie in $S^{n-1}$.

Corollary. $R^{s} \cap S^{n-1}=\varnothing$.

Proof. $R^{s} \subset \bigcap_{\sigma=0}^{i} \pi\left(p_{\sigma}\right)$ implies that $\widetilde{P}^{i} \subset \pi(p) \cap S^{n-1}$ for any $p \in$ $R^{s} \cap S^{n-1}$. But $R^{s} \cap \widetilde{P}^{i}=\varnothing$ and 2.3.3 Corollary 1 imply that this is not possible. Thus $R^{s} \cap S^{n-1}=\varnothing$.

Let $n \geqslant 2 i+2$. Thus there is a point $r \in R^{s}$. By 2.6.4(1), $P^{2 k+2}=$ $\left[p_{0}, \ldots, p_{k}, r, q_{0}, \ldots, q_{k}\right]$ is a $(2 k+2)$-flat; $0 \leqslant k \leqslant i$.

2.6.6. LеммA. $P^{2 k+2} \cap S^{n-1}$ is a nondegenerate $S^{2 k+1}$ with the index $k ; 0 \leqslant k \leqslant i$.

Proof. Obviously, $P^{2}=\left[p_{0}, q_{0}, r\right] \subset P^{2 k+2}$. Since $\left[p_{0}, q_{0}\right] \not \subset S^{n-1}$ and $r \in \pi\left(p_{0}\right) \cap \pi\left(q_{0}\right), r \notin S^{n-1}$ implies that $P^{2} \cap S^{n-1}$ is an $S^{1}$ by 2.1.5. Hence, $P^{2 k+2} \cap S^{n-1}$ is an $S^{2 k+1}$ with the tangent hyperplane $\pi(p) \cap P^{2 k+2}$ at $p \in S^{2 k+1}$. Furthermore, $\left[p_{\alpha}, q_{\alpha}\right] \not S^{2 k+1}$ implies that $S^{2 k+1}$ is differentiable at each of the points $p_{\alpha}, q_{\alpha} ; \alpha=0, \ldots, k$.

By 2.6.4(3) and 2.6.4(4),

and

$$
\pi\left(p_{\alpha}\right) \cap P^{2 k+2}=\left[p_{0}, \ldots, p_{k}, r, q_{0}, \ldots, \hat{q}_{\alpha}, \ldots, q_{k}\right]
$$

$$
\pi\left(q_{\alpha}\right) \cap P^{2 k+2}=\left[q_{0}, \ldots, q_{k}, r, p_{0}, \ldots, \hat{p}_{\alpha}, \ldots, p_{k}\right] ; \alpha=0, \ldots, k .
$$

Therefore, $\bigcap_{\alpha=0}^{k}\left(\pi\left(p_{\alpha}\right) \cap \pi\left(q_{\alpha}\right) \cap P^{2 k+2}\right)=\{r\}$. Since $r \notin S^{n-1}, S^{2 k+1}$ is nondegenerate by 2.2.10 Corollary.

By 2.4 .4 , ind $S^{2 k+1} \leqslant k$. As $\left[p_{0}, \ldots, p_{k}\right] \subset P^{2 k+2} \cap \widetilde{P}^{i} \subseteq P^{2 k+2} \cap$ $S^{n-1}=S^{2 k+1}$, we obtain ind $S^{2 k+1}=k$.

The preceding lemma is readily extended to the case $n=2 i+1$ if we assume that $0 \leqslant k \leqslant i-1$. Then by $2.6 .3, R^{1}=\bigcap_{\sigma=0}^{i-1}\left(\pi\left(p_{\sigma}\right) \cap \pi\left(q_{\sigma}\right)\right)$ and $R^{1} \cap S^{n-1}=\left\{p_{i}, q_{i}\right\}$. We then choose $r \in R^{1} \backslash S^{n-1}$ and our result is valid for $P^{2 k+2}=\left[p_{0}, \ldots, p_{k}, r, q_{0}, \ldots, q_{k}\right] ; 0 \leqslant k \leqslant i-1$.

In summary, we have

2.6.7. THEOREM. Let $S^{n-1}$ be nondegenerate; ind $S^{n-1}=i \geqslant 1$ and $n \geqslant 4$. Let $0 \leqslant k \leqslant i-1[0 \leqslant k \leqslant i-2]$ and let $r \in R^{s}\left[r \in R^{1} \backslash S^{n-1}\right]$ when $n>2 i+1[n=2 i+1]$. Then $P^{n}=\left[R^{n-2(k+1)}, P^{2(k+1)}\right]$ where

(1) $R^{n-2(k+1)}=\bigcap_{\sigma=0}^{k}\left(\pi\left(p_{\sigma}\right) \cap \pi\left(q_{\sigma}\right)\right)$,

(2) $P^{2(k+1)}=\left[p_{0}, \ldots, p_{k}, r, q_{0}, \ldots, q_{k}\right]$,

(3) $R^{n-2(k+1)} \cap P^{2(k+1)}=\{r\}$,

(4) $R^{n-2(k+1)} \cap S^{n-1}$ is a nondegenerate $S^{n-2 k-3}$ of index $i-(k+1)$, and 
(5) $P^{2(k+1)} \cap S^{n-1}$ is a nondegenerate $S^{2 k+1}$ of index $k$.

3. Linearly connected sets. In this chapter, we shall prove that a nondegenerate $S^{n-1} \subset P^{n}$ with a positive index is the boundary of a certain type of linearly connected set as introduced in [3]. Marchaud's results then imply that such an $S^{n-1}$ is a quadric.

\subsection{Introduction.}

3.1.1. A set $M$ in $P^{n}$ is linearly connected if $P^{1} \cap M$ is connected for all $P^{1} \subset P^{n}$.

Thus $M$ is connected and $P^{n} \backslash M$ is linearly connected.

3.1.2. Let $M_{\sigma}$ be sets in $P^{k} ; k \geqslant 2 ; \sigma=1,2 . M_{1}$ and $M_{2}$ are a linearly connected pair in $P^{k}$ if $M_{1}$ and $M_{2}$ are nonvoid, open, disjoint, linearly connected sets such that $P^{k} \backslash\left(M_{1} \cup M_{2}\right)=\bar{M}_{1} \cap \bar{M}_{2}$.

Let $n \geqslant 3$. Let $A$ and $B$ be a linearly connected pair in $P^{n}$. Let $F=\bar{A} \cap$ $\bar{B}$. Then $P^{n}=A \cup B \cup F, \bar{A} \cap B=\bar{B} \cap A=\varnothing, \bar{B}=B \cup F$ and $\bar{A}=A \cup F$.

We collect some definitions and results regarding such linearly connected sets; cf. [3] .

3.1.3. Let $P^{k} \subset P^{n}$ be a $k$-flat. $P^{k}$ is a secant if $P^{k} \cap A \neq \varnothing \neq P^{k} \cap B$. $P^{k}$ supports $A[B]$ if $P^{k} \subset \bar{B}[\bar{A}], P^{k} \cap F \neq \varnothing$ and $P^{k} \cap B \neq \varnothing\left[P^{k} \cap A \neq \varnothing\right]$.

3.1.4. Let $P^{k} \subset P^{n}$ be a secant. Then $P^{k} \cap A$ and $P^{k} \cap B$ are a linearly connected pair in $P^{k}$ with the common boundary $P^{k} \cap F$. In particular, a line is a secant if and only if it meets $F$ in exactly two distinct points.

3.1.5. A point $p$ in $F$ is regular if there exists a secant line through $p$. Otherwise, $p$ is an irregular point of $F$.

3.1.6. The index of $A[B]$ is the maximum dimension of any flat of $P^{n}$ contained in $A[B]$. We shall denote the indices by $i_{m} A$ and $i_{m} B$ respectively.

3.1.7. A set $O$ in $P^{2}$ is an oval if $O$ is an injective continuous image of $P^{1}$ into $P^{2}$ and $O$ is the common boundary of a linearly connected pair in $P^{2}$.

3.1.8. A oval $O$ has a paratingent at each point $p$; i.e. a line of accumulation of lines through two distinct points of $O$ which tend simultaneously to $p$.

Let $F$ be regular; that is, every $p \in F$ is regular.

3.1.9. Let $P^{2} \subset P^{n}$ be a secant. Then $P^{2} \cap F$ is either a pair of lines or an oval. Thus, any line which meets $F$ in three distinct points is contained in $F$.

3.1.10. Let $c \in A[B]$. Then there is a $k$-flat through $c$ in $A[B]$ where $k=i_{m} A\left[i_{m} B\right]$.

3.1.11. Let $\min \left\{i_{m} A, i_{m} B\right\}>0$. Then a line is a paratingent of an oval if and only if it meets the oval at exactly one point.

3.1.12. Let $F$ be regular. If $\min \left\{i_{m} A, i_{m} B\right\}>0$, then $F$ is a nondegenerate ruled quadric.

3.2. Linearly connected pairs. Let $A$ and $B$ be a linearly connected pair 
in $P^{n}$ such that

$$
\text { (1) } F=P^{n} \backslash(A \cup B)=\bar{A} \cap \bar{B} \text {, (2) } F \text { is regular, }
$$

(3) $\min \left\{i_{m} A, i_{m} B\right\} \geqslant 1$.

We shall show that $F$ is a hypersurface of order 2 in $P^{n} ; n \geqslant 3$.

3.2.1. Lemma. Let $F$ satisfy (1) $)_{n}$. Then $F$ is a hypersurface in $P^{n}$.

Proof. Clearly, $F$ is closed.

Let $p \in F$. Since $p$ is regular, there exists a secant $L=[p, q]$ such that $L \cap F=\{p, q\}$. Thus, there is a point $a_{0} \in L \cap A$ and a point $b_{0} \in L \cap B$.

Choose a hyperplane $P^{n-1}$ through $a_{0}$ such that $L \cap P^{n-1}=\left\{a_{0}\right\}$. Thus, there is an open $(n-1)$-ball $N_{0}$ about $a_{0}$ in $P^{n-1} \cap A$.

If $a \in N_{0}$, the secant $L_{a}=\left[b_{0}, a\right]$ meets $F$ in exactly two points. Let $\left\{a_{\sigma}\right\}$ be a sequence of points in $N_{0}$ with the limit point $a_{0}, L_{a_{0}}=\left[b_{0}, a_{\sigma}\right]$. Then $\lim L_{a_{o}}=\left[b_{0}, a_{0}\right]=[p, q]$.

Let $U$ be an open neighbourhood of $p$ in $F$ such that $q \notin \bar{U}$. For $a_{\sigma}$ sufficiently close to $a_{0}, L_{a_{\sigma}}$ meets $U$ in exactly one point. Thus, there exists an $(n-1)$-ball $N_{1}$ about $a_{0}$ in $N_{0}$ such that $L_{a} \cap U$ is a point for all $a \in N_{1}$.

Let $U_{1}=\left\{u \in U \mid\{u\}=L_{a} \cap U\right.$ where $\left.a \in N_{1}\right\}$.

Since $a_{0} \in N_{1}, p \in U_{1}$. Suppose that $\{u\}=L_{a_{1}} \cap U=L_{a_{2}} \cap U$ for $u \in U_{1}$ and $a_{1} \neq a_{2}$ in $N_{1}$. Then $\left\{a_{1}, a_{2}\right\} \subset N_{1} \subset N_{0} \subset P^{n-1}$ implies that $b_{0} \in\left[u, a_{1}\right]=\left[a_{1}, a_{2}\right] \subset P^{n-1} ; \mathrm{a}$ contradiction. Hence, the correspondence between $a \in N_{1}$ and $L_{a} \cap U \in U_{1}$ is a bijection. Obviously, it is a homeomorphism.

Since $p$ is arbitrary, the lemma follows.

Corollary. Let $P^{k} \subset P^{n}$ be a secant; $3 \leqslant k \leqslant n-1$. Then $F_{k}=$ $P^{k} \cap F$ is a hypersurface in $P^{k}$.

Proof. If $F_{k}$ is regular, then the result follows by 3.2.1.

Let $v_{1} \neq v_{2}$ be points in $V$, the set of irregular points of $F_{k}$. The line $\left[v_{1}, v_{2}\right]$ meets $F_{k}$ at a third point by 3.1.4 and 3.1.5. Since $F_{k} \subset F, 3.1 .9$ implies that $\left[v_{1}, v_{2}\right] \subset F_{k}$. Hence $V$ is a flat and clearly, $\operatorname{dim} V \leqslant k-2$.

From the proof of 3.2.1, $F_{k} \backslash V$ is locally homeomorphic to the union of a finite number of open $(k-1)$-balls.

3.2.2. Lemma. Let $F \subset P^{3}$ satisfy (1) $)_{3}$. Then every $P^{2} \subset P^{3}$ is a secant.

Proof. By (1) $)_{3}$, there is a line $L_{A} \subset A$ and a line $L_{B} \subset B$. Hence, $P^{2} \cap L_{A} \neq \varnothing \neq P^{2} \cap L_{B}$ for all $P^{2} \subset P^{3}$.

3.2.3. Lемма. Under the hypothesis of 3.2.2, let $P^{2} \cap F$ be an oval $O$. Then $O$ is an $S^{1}$. 
Proof. Since $F$ is regular, every line meets $O$ in at most two distinct points. Therefore, $O$ is an $S^{1}$ if $O$ has a unique tangent at each point.

Let $p \in O$. By 3.1.8 and 3.1.11, there is a paratingent $T$ of $O$ at $p$ and $T \cap O=\{p\}$. Thus $T$ is not a secant and by 3.1.3, $T$ supports $A$ or $B$. We may assume $T \subset \bar{B}$; thus, $T \cap A=\varnothing$.

Let $q \in O \backslash\{p\}$. Since $[p, q] \cap O=\{p, q\}, L=[p, q]$ is a secant and $L=[a, b]$ where $a \in L \cap A$ and $b \in L \cap B$. By 3.1.10, there is a line $L_{a} \subset A$ through $a$. Since $T \cap A=\varnothing$, we have $\left[L_{a}, L\right] \neq[T, L]=P^{2}$.

Obviously, $\left[L_{a}, L\right]$ is a secant. By 3.1.9, $L_{a} \cap F=\varnothing$ implies that $\left[L_{a}, L\right]$ $\cap F$ is an oval $O^{\prime}$. Then $\{p, q\} \subset O^{\prime}$ and $O^{\prime}$ has a paratingent $T^{\prime}$ at $p$. Since $T^{\prime} \cap O^{\prime}=\{p\}$ and $T^{\prime} \cap L_{a} \in A, T^{\prime} \subset \bar{A}$ by 3.1.3. As $T \subset \bar{B}$, this implies that $P_{0}^{2}=\left[T, T^{\prime}\right]$ is a plane.

Again, $P_{0}^{2}$ is a secant through $p$. It is immediate that $i_{m}\left(P^{2} \cap A\right)=$ $i_{m}\left(P^{2} \cap B\right)=0$. Thus, $P_{0}^{2} \cap F$ is a pair of lines $L_{1}$ and $L_{2}$ through $p$ by 3.1.9.

Since $p \in O$ is arbitrary, there are two distinct lines of $F$ through each point of $O$. Since every plane is a secant, there are exactly two such lines through each point of $O$ by 3.1.9.

Let $T^{\prime \prime} \subset P^{2}$ be a paratingent of $O$ at $p$. Since $T \subset \bar{B}, T^{\prime \prime} \subset \bar{B}$ as well. From the preceding, $\left[T^{\prime \prime}, T^{\prime}\right] \cap F$ is a pair of distinct lines through $p$. Since these must be $L_{1}$ and $L_{2}$, we have

$$
T^{\prime \prime}=P^{2} \cap\left[T^{\prime \prime}, T^{\prime}\right]=P^{2} \cap\left[L_{1}, L_{2}\right]=P^{2} \cap\left[T, T^{\prime}\right]=T .
$$

Therefore, $O$ has a unique tangent at each point $p$.

3.2.4. THEOREM. Let $F \subset P^{3}$ satisfy (1) $)_{3}$. Then $F$ is a nondegenerate $S^{2}$ with index 1 .

Proof. By 3.2.2, 3.2.3 and 3.1.9, $P^{2} \cap F$ is either a pair of lines or an $S^{1}$ for all $P^{2} \subset P^{3}$. As there is a line $L \subset A, P^{2} \cap F$ is an $S^{1}$ for all $P^{2}$ through $L$. By 3.2.1 and 2.1.4, $F$ is an $S^{2}$.

Obviously, $F$ is nondegenerate and from the proof of $3.2 .3, F$ has the index 1 .

Let $F \subset P^{n}$ satisfy $(1)_{n} ; n \geqslant 4$.

3.2.5. LEMMA. If $P^{k}$ is not a secant, then $P^{k} \cap F$ is a flat; $-1 \leqslant k \leqslant n-1$.

Proof. We may assume that $\operatorname{dim}\left(P^{k} \cap F\right)>0$. By 3.1.3, we may assume that $P^{k} \cap A=\varnothing$ say.

Let $p \neq q$ in $P^{k} \cap F$. Since $[p, q] \cap A=\varnothing,[p, q]$ is not a secant. By 3.1.4 and 3.1.9, $[p, q] \subset F$.

3.2.6. LEMma. Let $P^{2} \subset P^{n}$. Then $P^{2} \cap F$ is a flat or a pair of lines or an $S^{1}$. 
Proof. By 3.2.5 and 3.1.9, we may assume that $P^{2} \cap F$ is an oval $O$. Obviously, there is a line $L$ contained in either $P^{2} \cap A$ or $P^{2} \cap B$. Let $L \subset$ $P^{2} \cap B$. Since $P^{2}$ is a secant, there is a point $a \in P^{2} \cap A$. As $i_{m} A \geqslant 1,3.1 .10$ implies that there is a line $L^{\prime} \subset A$ through $a$. Since $A \cap B=\varnothing, P^{3}=\left[L, L^{\prime}\right]$ is a 3-flat. Clearly, $P^{3}$ is a secant and $P^{2} \subset P^{3}$.

By 3.1.4, $A_{3}=P^{3} \cap A$ and $B_{3}=P^{3} \cap B$ are a linearly connected pair in $P^{3}$ with $F_{3}=P^{3} \cap F=\bar{A}_{3} \cap \bar{B}_{3}$. As $L \subset B_{3}$ and $L^{\prime} \subset A_{3}$, we have $\min \left\{i_{m} A_{3}, i_{m} B_{3}\right\}>0$. Then every plane in $P^{3}$ is a secant and it is immediate that $F_{3}$ is regular. Thus, $F_{3}$ is a nondegenerate $S^{2}$ with the index 1 by 3.2.4. In particular, $P^{2} \cap S^{2}=P^{2} \cap F=O$ is an $S^{1}$ by 2.1.5.

3.2.7. Lemma (MARchaud [3]). Let $P^{k} \subset P^{n}$ be a secant such that $i_{m}\left(P^{k} \cap A\right)=i_{m}\left(P^{k} \cap B\right)=0 ; 1 \leqslant k \leqslant n-1$. Then $P^{k} \cap F$ is a pair of $(k-1)$-flats.

3.2.8. LEMmA. Let $P^{k} \subseteq P^{n} ; 2 \leqslant k \leqslant n$. Then $P^{k} \cap F$ is a flat or a pair of $(k-1)$-flats or an $S^{k-1}$.

Proof. By 3.2.6, the assertion is true for $k=2$. Suppose it has been proven up to $k-1$. By 3.2.5 and 3.2.7, we may assume that $P^{k}$ is a secant with $i_{m}\left(P^{k} \cap B\right)>0$. Thus, there is a line $L \subset P^{k} \cap B$ and a point $a \in P^{k} \cap A$. Clearly, we wish to show that $P^{k} \cap F$ satisfies 2.1.4.

By the induction hypothesis, $P^{k-1} \cap F$ is a flat or a pair of $(k-2)$-flats or an $S^{k-2}$ for all $P^{k-1} \subset P^{k}$. As $L \subset P^{k} \cap B$ and $L \cap F=\varnothing$, this implies that every $(k-1)$-section of $P^{k} \cap F$ is either degenerate or nondegenerate; cf. 2.1.3.

Obviously, the plane $P^{2}=[L, a]$ is a secant and $P^{2} \cap F$ is an $S^{1}$ by 3.1.9 and 3.2.6. Let $P^{k-1} \subset P^{k}$ contain $P^{2}$. Then $S^{1} \subset P^{k-1} \cap F$ implies that $P^{k-1} \cap F$ is an $S^{k-2}$. By 3.2.1 Corollary and 2.1.4, $P^{k} \cap F$ is an $S^{k-1}$.

3.2.9. THEOREM. Let $n \geqslant 3$. If $F$ satisfies $(1)_{n}$, then $F$ is a nondegenerate $S^{n-1}$ with ind $S^{n-1} \geqslant 1$.

Proof. As $\min \left\{i_{m} A, i_{m} B\right\} \geqslant 1, F$ is an $S^{n-1}$ by 3.2.8. As in 3.2.6, we construct a $P^{2}$ such that $P^{2} \cap F$ is an $S^{1}$ and $P^{3}$ through $P^{2}$ such that $P^{3} \cap F$ is a nondegenerate $S^{2}$ with ind $S^{2}=1$. Then ind $F \geqslant$ ind $S^{2}=1$. As $F$ is regular, $F$ is nondegenerate by 2.2 .10 .

3.3. Nondegenerate $S^{n-1}$. Let $S^{n-1}$ be nondegenerate with ind $S^{n-1}=$ $i>0 ; n \geqslant 3$. We shall prove that $F=S^{n-1}$ satisfies $(1)_{n} ;$ cf. 3.2.

3.3.1. Let $\left\{c, c^{\prime}\right\} \subset P^{n} \backslash S^{n-1}$. We define $c \sim c^{\prime}$ if $\left[c, c^{\prime}\right] \cap S^{n-1}$ is void or a point or a pair of distinct points $p_{1}$ and $p_{2}$ such that $c$ and $c^{\prime}$ lie on the same line segment bounded by $p_{1}$ and $p_{2}$. 
3.3.2. Clearly, $\sim$ is both reflexive and symmetric. It is easy to verify that it is, in fact, an equivalence relation on $P^{n} \backslash S^{n-1}$.

Let $A$ be an equivalence class [ -class] of $P^{n} \backslash S^{n-1}$. Since there is a line meeting $S^{n-1}$ in exactly two distinct points, there exists a second $\sim$-class of $P^{n} \backslash S^{n-1}$, say $B$, by 3.3.1. For any $P^{2} \subset P^{n}$, it is immediate that

$$
P^{2}=\left(P^{2} \cap A\right) \cup\left(P^{2} \cap S^{n-1}\right) \cup\left(P^{2} \cap B\right)
$$

and thus, $A$ and $B$ are the only two such classes. Hence, $A \cup B=P^{n} \backslash S^{n-1}$ and $A \cap B=\varnothing$.

3.3.3. Let $P^{1} \subset P^{n}$. By 3.3.1, $P^{1} \cap A\left[P^{1} \cap B\right]$ is a flat or an open segment. Hence, $A$ and $B$ are linearly connected sets by 3.1.1.

Obviously, $\bar{A}=A \cup S^{n-1}=P^{n} \backslash B$ and $\bar{B}=B \cup S^{n-1}=P^{n} \backslash A$. Thus $A \cap B=\varnothing$ implies that $S^{n-1}=\bar{A} \cap \bar{B}$. By 2.2.10, $S^{n-1}$ is regular.

In conclusion, we have

3.3.4. THEOREM. Let $S^{n-1}$ be nondegenerate with ind $S^{n-1}>0 ; n \geqslant 3$. Then $S^{n-1}=P^{n} \backslash(A \cup B)=\bar{A} \cap \bar{B}$ where $A$ and $B$ are a linearly connected pair in $P^{n}$. Moreover, $S^{n-1}$ is regular.

It remains to show that $\min \left\{i_{m} A, i_{m} B\right\} \geqslant 1$. This is true when $n=3$, as a nondegenerate $S^{2}$ with ind $S^{2}=1$ is a quadric by 2.4.5.

3.3.5. LEMMA. Under the hypothesis of 3.3.4, there is a 3-flat $P^{3}$ such that $P^{3} \cap S^{n-1}$ is a nondegenerate $S^{2}$ with ind $S^{2}=1$.

Proof. We may assume that $n \geqslant 4$. Since $S^{n-1}$ is nondegenerate, there is a $P^{2}$ such that $P^{2} \cap S^{n-1}$ is an $S^{1}$. Let $\left\{p_{0}, p_{1}\right\} \subset S^{1} ; p_{0} \neq p_{1}$. Let $P_{k}^{1}$ be the tangent of $S^{1}$ at $p_{k}, k=0,1$. By 1.3.3, $P_{k}^{1} \cap S^{1}=\left\{p_{k}\right\}$ and $P_{0}^{1} \cap P_{1}^{1}$ is a point $r \notin S^{n-1}$.

By 2.6.2, $R^{n-2}=\pi\left(p_{0}\right) \cap \pi\left(p_{1}\right)$ is an $(n-2)$-flat and $R^{n-2} \cap S^{n-1}$ is a nondegenerate $S^{n-3}$ with ind $S^{n-3}=i-1 \geqslant 0$. Obviously, $r \in R^{n-2} \backslash S^{n-3}$. Let $p \in S^{n-3}$.

Since $S^{n-3}$ is differentiable at $p$, there is a point $p^{\prime} \in S^{n-1}$ such that $\left[p, p^{\prime}\right] \not \subset S^{n-3}$. Whether $\left[r, p, p^{\prime}\right]$ is a plane or a line, there is a $P^{1}$ through $r$ intersecting $S^{n-3}$ at distinct points $u_{1}$ and $u_{2}$. Then $P^{1}=\left[r, u_{1}, u_{2}\right] \not \subset S^{n-1}$ and $P^{1} \subset R^{n-2}$.

By 2.2.4, $u_{\sigma} \in S^{n-3}$ implies that $\left[u_{\sigma}, p_{0}\right] \subset S^{n-1}, \sigma=1,2$. Thus $P^{1} \cap$ $P^{2}=\{r\}$ and $P^{3}=\left[P^{2}, P^{1}\right]$ is a 3-flat. Since $S^{1} \subset P^{3} \cap S^{n-1}$; by 2.1.5, $P^{3} \cap$ $S^{n-1}$ is an $S^{2}$. Hence, $\left[u_{\sigma}, p_{0}\right] \subset S^{2}$ and ind $S^{2}=1$.

Since $p_{0} \in S^{1} \subset S^{2}, p_{0}$ is a differentiable point of $S^{2}$ with the tangent plane $\tilde{\pi}\left(p_{0}\right)=\pi\left(p_{0}\right) \cap P^{3}$. Since $P^{1}=\left[u_{1}, u_{2}\right] \not \subset S^{2},\left[p_{0}, u_{1}\right] \neq\left[p_{0}, u_{2}\right]$ and thus, $\tilde{\pi}\left(p_{0}\right) \cap S^{2}=\left[p_{0}, u_{1}\right] \cup\left[p_{0}, u_{2}\right]$ by 2.3.6. Hence, $S^{2}$ is nondegenerate by 2.3.3 Corollary 1 . 
3.3.6. Lemma. Under the hypothesis of 3.3.4, $\min \left\{i_{m} A, i_{m} B\right\} \geqslant 1$.

Proof. By 3.3.5, there is a $P^{3}$ such that $P^{3} \cap S^{n-1}$ is a nondegenerate $S^{2}$ with ind $S^{2}=1$. By 3.3.4,

$$
S^{2}=P^{3} \backslash\left(A_{3} \cup B_{3}\right)=\bar{A}_{3} \cap \bar{B}_{3},
$$

where $A_{3}$ and $B_{3}$ are a linearly connected pair in $P^{3}$. As $S^{2}$ is a quadric, this yields that $i_{m} A_{3}=i_{m} B_{3}=1$.

By 3.3.4, $P^{n}=A \cup S^{n-1} \cup B$ and hence,

$$
P^{3}=\left(P^{3} \cap A\right) \cup\left(P^{3} \cap S^{n-1}\right) \cup\left(P^{3} \cap B\right),
$$

where $P^{3} \cap A$ and $P^{3} \cap B$ are open, disjoint, linearly connected and thus, connected. Since $S^{2}$ is a quadric, $P^{3} \backslash S^{2}$ is the union of precisely two nonvoid connected sets. Thus, $P^{3} \cap A \neq \varnothing \neq P^{3} \cap B$ and (1) and (2) imply, for example, $P^{3} \cap A=A_{3}$ and $P^{3} \cap B=B_{3}$. Since $A_{3} \subset A$ and $B_{3} \subset B$, we have $i_{m} A \geqslant$ $i_{m} A_{3}$ and $i_{m} B \geqslant i_{m} B$.

Thus we obtain by 3.1 .12 ,

3.3.7. THEOREM. Let $n \geqslant 3$. An $S^{n-1} \subset P^{n}$ is nondegenerate with ind $S^{n-1} \geqslant 1$ if and only if $F=S^{n-1}$ satisfies (1) in 3.2; moreover, such an $S^{n-1}$ is a quadric.

Appendix: Quadrics. We shall now prove, independently of the concept of linear connectedness, that a nondegenerate $S^{n-1} \subset P^{n}$ with a positive index is a quadric.

Let $u_{\sigma} \equiv\left(\delta_{\sigma 0}, \delta_{\sigma 1}, \ldots, \delta_{\sigma n}\right)$ be the base points of a (homogeneous) coordinate system of $P^{n} ; \sigma=0,1, \ldots, n$. Let $\mathbf{R}$ be the set of real numbers.

A quadric $Q^{n-1} P^{n}$ is given by an equation

$$
\sum_{\sigma, \mu=0}^{n} a_{\sigma \mu} x_{\sigma} x_{\mu}=0
$$

where $P^{0} \equiv\left(x_{0}, \ldots, x_{n}\right) \in P^{n}$ and $a_{\sigma \mu}=a_{\mu \sigma} ; \sigma, \mu=0,1, \ldots, n$.

$A Q^{n-1}$ is nondegenerate if $\operatorname{det}\left(a_{\sigma \mu}\right) \neq 0$ where $\left(a_{\sigma \mu}\right)$ is the matrix of coefficients in (1). Finally, if $Q^{n-1}$ is nondegenerate, then the tangent hyperplane $\omega(p)$ of $Q^{n-1}$ exists at each point $p$ in $Q^{n-1}$.

\section{A.1. Preparatory lemmas.}

A.1.1. Lemma. Let $S^{n-1}$ be nondegenerate; $n \geqslant 4$, ind $S^{n-1}=i \geqslant 1$. Let $P^{k} \cap S^{n-1}=S^{k-1}$ have the index $0 ; 2 \leqslant k \leqslant \min \{n-2, n-i+1\}$. Then there is a $P^{k+1}$ through $P^{k}$ such that $P^{k+1} \cap S^{n-1}$ is a nondegenerate $S^{k}$ with the index 1 .

Proof. Since ind $S^{k-1}=0$ and $k \geqslant 2$, there is a $P^{2} \subset P^{k}$ such that 
$P^{2} \cap S^{k-1}$ is an $S^{1}$. Then there is a $P^{3}$ through $P^{2}$ such that $P^{3} \cap S^{n-1}$ is a nondegenerate $S^{2}$ with the index 1 ; cf. the proof of 3.3.5.

Clearly, $P^{3} \cap P^{k}=P^{2}$ and $P^{k+1}=\left[P^{k}, P^{3}\right]$ is a $(k+1)$-flat. By 2.1.5, $P^{k+1} \cap S^{n-1}$ is necessarily an $S^{k}$. As ind $S^{2}=1$ [ind $S^{k-1}=0$ ], we have ind $S^{k} \geqslant 1$ [ind $S^{k} \leqslant 1$ ]. Hence, ind $S^{k}=1$. Now $S^{k}$ is clearly nondegenerate.

The following assertions are obvious.

A.1.2. Lemma. Let ind $S^{n-1}=0 ; n \geqslant 3$. Choose $p_{\sigma} \in S^{n-1}$ such that $P^{n}=\left[p_{0}, \ldots, p_{n}\right]$. If $S^{n-1}$ is a quadric, then $\bigcap_{\sigma=0}^{n} \pi\left(p_{\sigma}\right)=\varnothing$.

Corollary. $\bigcap_{\sigma=0 ; \sigma \neq k}^{n} \pi\left(p_{\sigma}\right)=P(k) \not \subset\left[p_{0}, \ldots, \hat{p}_{k}, \ldots, p_{n}\right] ; k=0$, $1, \ldots, n$.

A.2. Nondegenerate $S^{n-1}$ with index 1 . In order to prove our theorem, we shall construct a set in $S^{n-1}$ and coordinates in $P^{n}$ for every $i$. Then we show that there is a unique nondegenerate quadric containing this set and that this quadric is identical with $S^{n-1}$.

In the following sections, we deal with special values of $i$.

A.2.1. By 2.4.5, a nondegenerate $S^{2} \subset P^{3}$ with the index 1 is a quadric. Assume that every $S^{k-1} \subset P^{k}$ with ind $S^{k-1}=1$ is a quadric; $3 \leqslant k \leqslant n-1$. Let $S^{n-1} \subset P^{n}$ be nondegenerate with ind $S^{n-1}=1 ; n \geqslant 4$.

By 2.6.3, there are points $p_{0}, q_{0}$ in $S^{n-1} ;\left[p_{0}, q_{0}\right] \not \subset S^{n-1}$. Then $R^{n-2}$ $=\pi\left(p_{0}\right) \cap \pi\left(q_{0}\right)$ is an $(n-2)$-flat and $R^{n-2} \cap S^{n-1}$ is an $S^{n-3}$ of index 0 .

Choose $n-2$ points $r_{\sigma} \in S^{n-3}$ such that $R^{n-3}=\left[r_{0}, \ldots, r_{n-3}\right] \subset$ $R^{n-2}$ is an $(n-3)$-flat. We assume $\left\{p_{1}, q_{1}\right\} \subset\left\{r_{0}, \ldots, r_{n-3}\right\}$; cf. 2.6.3. By A.1.1 and the induction hypothesis, $S^{n-3}$ is a quadric. Thus $\bigcap_{\sigma=0}^{n-3}\left(\pi\left(r_{\sigma}\right) \cap R^{n-2}\right)$ is a point $r_{n-2} \notin R^{n-3}$, by A.1.2 Corollary. Clearly, $R^{n-2}=\left[R^{n-3}, r_{n-2}\right]$ and $r_{n-2} \in R^{s} ;$ cf. 2.6.4. Hence $r_{n-2} \notin S^{n-1}$.

From 2.6.7 with $r=r_{n-2}$ and $k=0$, we obtain $P^{n}=\left[R^{n-2}, P^{2}\right]$ where $P^{2}=\left[p_{0}, q_{0}, r_{n-2}\right], R^{n-2} \cap P^{2}=\left\{r_{n-2}\right\}$ and $P^{2} \cap S^{n-1}$ is an $S^{1}$. Thus, $P^{n}=\left[r_{0}, \ldots, r_{n-2}, r_{n-1}, r_{n}\right]$ where $r_{n-1}=p_{0}, r_{n}=q_{0}$. By A.1.1 and 2.4.5, $S^{1}$ is a conic.

Finally, we observe

A.2.2. LemmA. (1) $P^{2} \subset \pi\left(r_{\sigma}\right) ; \sigma=0,1, \ldots, n-3$; cf. 2.2.4.

(2) $R^{n-3} \subset \pi(p)$ for each $p \in S^{1}$.

A.2.3. Let $r_{\sigma}$ be the base points (of a coordinate system) of $P^{n} ; \sigma=0$, $\ldots, n$. Let $\mathrm{Q}^{n-1} \subset P^{n}$ be given by

$$
x_{n-2}^{2}+2 a_{n-1, n} x_{n-1} x_{n}+\sum_{\sigma, \mu=0 ; \sigma \neq \mu}^{n-3} a_{\sigma \mu} x_{\sigma} x_{\mu}=0 ; \quad \operatorname{det}\left(a_{\sigma \mu}\right) \neq 0 .
$$

Then $r_{n-2} \equiv(0, \ldots, 0,1,0,0) \notin Q^{n-1}$ and the tangent hyperplanes 
$\omega\left(r_{n-1}\right)$ and $\omega\left(r_{n}\right)$ are given by $x_{n}=0$ and $x_{n-1}=0$ respectively. Thus

$$
\begin{aligned}
\pi\left(r_{n-1}\right) \cap \pi\left(r_{n}\right) & =\left[r_{0}, \ldots, r_{n-2}\right]=R^{n-2}=\omega\left(r_{n-1}\right) \cap \omega\left(r_{n}\right), \\
\pi\left(r_{\sigma}\right) & =\left[R^{n-2}, r_{\sigma}\right]=\omega\left(r_{\sigma}\right) ; \quad \sigma=n-1, n .
\end{aligned}
$$

Since $S^{n-3}$ and $S^{1}$ are a quadric and conic, respectively, we choose $a_{\sigma \mu}$ satisfying (1) so that

$$
S^{n-3}=R^{n-2} \cap Q^{n-1} \text { and } S^{1}=P^{2} \cap Q^{n-1} .
$$

Then $\mathbf{Q}^{n-1}$ is uniquely determined.

As $\pi(p) \cap R^{n-2}=\omega(p) \cap R^{n-2}$ is the tangent hyperplane in $R^{n-2}$ at a point $p,(2)$ implies that

$$
\pi(p)=\left[\pi(p) \cap R^{n-2}, r_{n-1}, r_{n}\right]=\omega(p) \text { for } p \in S^{n-3} \text {. }
$$

Similarly, $\pi(p) \cap P^{2}=\omega(p) \cap P^{2}$ for $p \in S^{1}$ and thus

$$
\pi(p)=\left[R^{n-3}, \pi(p) \cap S^{n-1}\right]=\omega(p) \text { for all } p \in S^{1}
$$

Therefore

$$
R^{n-3}=\bigcap_{p \in S^{1}} \pi(p)
$$

By (2) and (3), $\pi\left(r_{\sigma}\right) \cap S^{n-1}$ and $\omega\left(r_{\sigma}\right) \cap Q^{n-1}$ are cones with the same vertex $r_{\sigma}$ and the same $(n-2)$-section $S^{n-3}$. Thus,

$$
\pi\left(r_{\sigma}\right) \cap S^{n-1}=\omega\left(r_{\sigma}\right) \cap Q^{n-1} ; \quad \sigma=n-1, n .
$$

Similarly,

$$
\left[p, R^{n-3}\right] \cap S^{n-1}=\left[p, R^{n-3}\right] \cap Q^{n-1} \text { for each } p \in S^{1} .
$$

A.2.4. LEMMA. $R^{n-3}=\pi(p) \cap \pi(q) \cap \pi(r)$ for any three mutually distinct points $p, q$ and $r$ in $S^{1}$.

Proof. Since $S^{1}$ is of order 2 , we have $P^{2}=[p, q, r]$ and $\pi(p) \cap \pi(q) \cap$ $\pi(r) \cap S^{1}=\varnothing$. Thus, $\operatorname{dim}(\pi(p) \cap \pi(q) \cap \pi(r)) \leqslant n-3$ and the result follows by A.2.3(6).

A.2.5. Lemma. Let $p \in S^{1}$. Then $\pi(p) \cap S^{n-1}=\pi(p) \cap Q^{n-1}$.

Proof. By 2.6.1, $\pi(p) \cap S^{n-1}$ is a 1-degenerate $S^{n-2}$ with the singular point $p$; ind $S^{n-2}=1$. Thus any line $L \subset S^{n-2}$ passes through $p$. By A.2.3(7), we may assume $p \neq r_{n-1}, r_{n}$. Then $p \notin \pi\left(r_{\sigma}\right)$ and thus, $\pi\left(r_{\sigma}\right) \cap L$ is a point $u_{\sigma} ; \sigma=n-1, n$. By A.2.3(7), $u_{\sigma} \in \mathrm{Q}^{n-1}$.

If $u_{n-1} \neq u_{n}$, then $L$ meets $Q^{n-1}$ at three mutually distinct points and thus, $L \subset \mathrm{Q}^{n-1}$; cf. A.2.3(3). If $u_{n-1}=u_{n}=u$, then by A.2.4, $u \in \pi\left(r_{n-1}\right) \cap$ 
$\pi\left(r_{n}\right) \cap \pi(p)=R^{n-3}$. Hence, $L=[p, u] \subset\left[p, R^{n-3}\right] \cap S^{n-1} \subset Q^{n-1}$ by A.2.3(8). Thus, $\pi(p) \cap S^{n-1} \subseteq \pi(p) \cap Q^{n-1}$.

The preceding argument is symmetric in $S^{n-1}$ and $Q^{n-1}$.

A.2.6. LemmA. Let $p \in S^{n-3} \backslash R^{n-3}$. Then $\pi(p) \cap S^{n-1}=\pi(p) \cap Q^{n-1}$.

Proof. As in A.2.5, we apply 2.6.1 and obtain $\pi(p) \cap S^{n-1}=S^{n-2}$. Let $L \subset S^{n-2}$ be a line. Thus $p \in L$. Since $S^{n-3}=R^{n-2} \cap S^{n-1}$ has the index 0 , this yields $L \cap R^{n-2}=\{p\}$. As $p \notin R^{n-3}$, we obtain $L \cap R^{n-3}=\varnothing$.

If $L$ meets $P^{2}$ at a point $q$, then $q \in S^{1}$ and by A.2.5, $L \subset \pi(q) \cap S^{n-1}$ $\subset Q^{n-1}$. Let $L \cap P^{2}=\varnothing$. Then for $q \in S^{1}, u(q)=\pi(q) \cap L$ is a point in $Q^{n-1}$. But A.2.4 and $L \cap R^{n-3}=\varnothing$ imply that $u=u(q)$ has at most two solutions in $S^{1}$ for any $u$. Hence, $L$ meets $Q^{n-1}$ in three mutually distinct points and thus, $L \subset \mathrm{Q}^{n-1}$. The lemma now follows; cf. the proof of A.2.5.

A.2.7. Theorem. Under the hypotheses of A.2.1 and A.2.3, $S^{n-1}=$ $Q^{n-1}$.

Proof. We first prove $Q^{n-1} \subseteq S^{n-1}$.

Let $L \subset Q^{n-1}$ be a line. By A.2.5, we assume that $L \cap P^{2}=\varnothing$. Thus, $u(q)=\pi(q) \cap L$ is a point in $S^{n-1}$ for each $q \in S^{1}$. Let $U=\left\{u(q) \mid q \in S^{1}\right\}$.

If $|U| \geqslant 3$, then $L \subset S^{n-1}$ by 2.1.5. If $U=\left\{u_{1}, u_{2}\right\}$, then say $u_{1} \in R^{n-3}$ by

A.2.4. Let $u_{2}=u(q)$. Then $L=\left[u_{1}, u_{2}\right] \subset\left[R^{n-3}, \pi(q) \cap L\right] \subset \pi(q)$ by A.2.3(5); a contradiction by assumption.

Let $U=\{u\}$. Then $u \in R^{n-3} \cap S^{n-1} \subset S^{n-3}$ by A.2.4. Observe that $u \in L \subset Q^{n-1}$ implies that $L \subset \omega(u)=\pi(u)$ by A.2.3(4).

Choose a point $p \in S^{n-3} \backslash R^{n-3}$. By A.2.6, $\pi(p) \cap L \subset S^{n-1}$. Since ind $S^{n-3}=0$ and $\{u, p\} \subset S^{n-3}, u \notin \pi(p)$ and thus, $L=[u, \pi(p) \cap L]$. By 2.2.4, $L \subset \pi(u)$ implies that $L \subset S^{n-1}$. Thus $Q^{n-1} \subseteq S^{n-1}$.

The preceding argument is symmetric in $S^{n-1}$ and $Q^{n-1}$.

A.3. Nondegenerate $S^{n-1}$ with ind $S^{n-1}=\left[\frac{1}{2}(n-1)\right] ; n$ even.

A.3.1. In this section, we prove that a nondegenerate $S^{2 i+1} \subset P^{2 i+2}$ of index $i$ is a quadric for $i>0$. By A.2, this assertion is true for $i=1$. We assume that it has been proven up to $i-1$.

Put $n=2 i+2$. Let $\left\{p_{\sigma}, q_{\sigma} \mid \sigma \in I=\{0,1, \ldots, i\} \subset S^{n-1}\right.$ satisfy 2.6.3. From 2.6.4(2), $R^{s}=\bigcap_{\sigma=0}^{i}\left(\pi\left(p_{\sigma}\right) \cap \pi\left(q_{\sigma}\right)\right)$ is an s-flat; $s=n-2(i+1)=0$. By 2.6.5 Corollary, $R^{s}$ is a point $r \notin S^{n-1}$.

We introduce the $i$-flats $\widetilde{P}^{i}, \widetilde{Q}^{i}, P_{\alpha}^{i}$ and $Q_{\alpha}^{i}$ as in 2.6.4; $\alpha \in I$. By 2.6.5, all of these $i$-flats are contained in $S^{n-1}$; moreover,

(1) $P^{n}=\left[p_{0}, \ldots, p_{i}, r, q_{0}, \ldots, q_{i}\right]=\left[\widetilde{P}^{i}, r, \widetilde{Q}^{i}\right]$, 
(2) $\pi\left(p_{\alpha}\right)=\left[\widetilde{P}^{i}, r, q_{0}, \ldots, \hat{q}_{\alpha}, \ldots, q_{i}\right], \alpha \in I$, and

(3) $\pi\left(q_{\alpha}\right)=\left[\widetilde{Q}^{i}, r, p_{0}, \ldots, \hat{p}_{\alpha}, \ldots, p_{i}\right], \alpha \in I$.

We apply 2.6.7 in the case $k=0$ and $R^{s}=\{r\}$. Then $P^{n}=\left[R^{n-2}, P^{2}\right]$ where $R^{n-2}=\pi\left(p_{0}\right) \cap \pi\left(q_{0}\right), P^{2}=\left[p_{0}, q_{0}, r\right], R^{n-2} \cap P^{2}=\{r\}, P^{2} \cap S^{n-1}$ is an $S^{1}$ and $R^{n-2} \cap S^{n-1}$ is a nondegenerate $S^{n-3}$; ind $S^{n-3}=i-1$. By the preceding,

$$
R^{n-2}=\pi\left(p_{0}\right) \cap \pi\left(q_{0}\right)=\left[p_{1}, \ldots, p_{i}, r, q_{1}, \ldots, q_{i}\right] .
$$

A.3.2. Choose the following base points $u_{k}$ of $P^{n}$ :

$$
u_{k}= \begin{cases}p_{k}, & k=0,1, \ldots, i, \\ r, & k=i+1, \\ q_{n-k}, & k=i+2, i+3, \ldots, n .\end{cases}
$$

Let $Q^{n-1} \subset P^{n}$ be given by

$$
x_{i+1}^{2}+2 \sum_{\sigma=0}^{i} a_{\sigma, n-\sigma} x_{\sigma} x_{n-\sigma}=0 ; \quad \operatorname{det}\left(a_{\sigma \mu}\right) \neq 0 .
$$

Clearly, $Q^{n-1}$ contains $\widetilde{P}^{i}, \widetilde{Q}^{i}, P_{\alpha}^{i}$ and $Q_{\alpha}^{i}, \alpha \in I$, and

$$
r=u_{i+1} \equiv\left(0, \ldots, 0, x_{i+1}, 0, \ldots, 0\right) \notin Q^{n-1} \quad\left(x_{i+1}=1\right) .
$$

For $\sigma=0, \ldots, n, \sigma \neq i+1,(1)$ implies that $\omega\left(u_{\sigma}\right)$ is given by $x_{n-\sigma}=0$.

Thus

$$
\omega\left(u_{\sigma}\right)=\left[u_{0}, \ldots, \hat{u}_{n-\sigma}, \ldots, u_{n}\right]=\pi\left(u_{\sigma}\right) \equiv x_{n-\sigma}=0 .
$$

By the induction hypothesis, $S^{n-3}=R^{n-2} \cap S^{n-1}$ is a quadric. Obviously, $S^{1}=P^{2} \cap S^{n-1}$ is a conic. Thus, we determine $Q^{n-1}$ uniquely by choosing $a_{\sigma \mu}$ in (1) so that

$$
S^{n-3}=R^{n-2} \cap Q^{n-1} \text { and } S^{1}=P^{2} \cap Q^{n-1} \text {. }
$$

A.3.3. LEMMA. $\pi\left(p_{\sigma}\right) \cap \pi\left(q_{\sigma}\right) \cap S^{n-1}=\pi\left(p_{\sigma}\right) \cap \pi\left(q_{\sigma}\right) \cap Q^{n-1} ; \sigma \in I$.

Proof. By A.3.2(3), we may assume $\sigma \neq 0$; e.g. $\sigma=i$. Let $j=i-1$. Clearly, $P^{n-2}=\pi\left(p_{i}\right) \cap \pi\left(q_{i}\right)$ is an $(n-2)$-flat and by $2.6 .2, \widetilde{S}^{n-3}=$ $p^{n-2} \cap S^{n-1}$ is a nondegenerate hypersurface of order 2 ; ind $\widetilde{S}^{n-3}=i-1$.

Using the coordinate system of A.3.2, $P^{n-2}$ is given by $x_{i}=x_{i+2}=0$ by A.3.2(2). Observe that $P^{n-2} \cap \widetilde{P}^{i}, P^{n-2} \cap \widetilde{Q}^{i}, P^{n-2} \cap P_{\alpha}^{i}$ and $P^{n-2} \cap$ $Q_{\alpha}^{i}$ are $j$-flats in $\widetilde{S}^{n-3} ; \alpha \in J=\Lambda\{i\}$. Finally, by the induction hypothesis, $\widetilde{S}^{n-3} \subset P^{n-2}$ is a quadric, say

(1) $\widetilde{S}^{n-3}=Q^{n-3} \equiv x_{i+1}^{2}+2 \sum_{\sigma=0}^{j} c_{\sigma, n-\sigma} x_{\sigma} x_{n-\sigma}=0, \quad x_{i}=x_{i+2}=0$. 
Let $\mu \in J$ and consider the plane $P_{\mu}^{2}=\left[p_{\mu}, q_{\mu}, r\right]$. Clearly, $P_{\mu}^{2} \cap S^{n-1}=$ $S_{\mu}^{1}$ is a nondegenerate curve of order 2 by 2.1.5. By A.1.2 and 2.4.5, $S_{\mu}^{1}$ is a conic.

Observe that $P_{0}^{2}=P^{2}$ (A.3.1) and thus, $S_{0}^{1}=S^{1} \subset \tilde{S}^{n-3}$. For $\mu \neq 0$, $S_{\mu}^{1} \subset S^{n-3} \cap \widetilde{S}^{n-3}$. As $S^{1} \cup S^{n-3} \subset Q^{n-1}$ and $\widetilde{S}^{n-3}=Q^{n-3}$, this implies that $S_{\mu}^{1} \subset Q^{n-1} \cap Q^{n-3}$ and in particular,

$$
S_{\mu}^{1}=P_{\mu}^{2} \cap Q^{n-1}=P_{\mu}^{2} \cap Q^{n-3} ; \quad \mu \in J .
$$

Then A.3.2(1), (1) and (2) imply that $c_{\mu, n-\mu}=a_{\mu, n-\mu}$ for $\mu \in J$. Thus, $Q^{n-3}$ $\subset Q^{n-1}$ and $P^{n-2} \cap S^{n-1}=\widetilde{S}^{n-3}=Q^{n-3}=P^{n-2} \cap Q^{n-1}$.

Corollary. $\pi\left(u_{\sigma}\right) \cap S^{n-1}=\pi\left(u_{\sigma}\right) \cap Q^{n-1} ; \sigma=0, \ldots, n, \sigma \neq i+1$.

Proof. Both $\pi\left(u_{\sigma}\right) \cap S^{n-1}$ and $\pi\left(u_{\sigma}\right) \cap Q^{n-1}$ are cones with the vertex $u_{\sigma}$ and the $(n-2)$-section $\pi\left(u_{\sigma}\right) \cap \pi\left(u_{n-\sigma}\right) \cap S^{n-1}$.

A.3.4. THEOREM. Under the hypotheses of A.3.1 and A.3.2, $S^{n-1}=$ $Q^{n-1}$.

Proof. We first show $Q^{n-1} \subseteq S^{n-1}$. Let $Q^{i} \subset Q^{n-1}$ be an $i$-flat. By A.3.3 Corollary, we may assume $\pi\left(u_{\sigma}\right) \cap Q^{i}$ is an $(i-1)$-flat in $S^{n-1} ; \sigma=0$, $\ldots, n, \sigma \neq i+1$. Then $\operatorname{dim} Q^{i}=i \geqslant 2$ and A.3.2(2) imply that there are at least three mutually distinct $\pi\left(u_{\sigma}\right) \cap Q^{i}$ 's. By $2.1 .5, Q^{i} \subset S^{n-1}$ and thus $\mathbf{Q}^{n-1} \subseteq S^{n-1}$.

The preceding argument is symmetric in $Q^{n-1}$ and $S^{n-1}$.

A.4. Nondegenerate $S^{n-1}$ with ind $S^{n-1}=i ; n=2 i+1 \geqslant 5$.

A.4.1. Put $n=2 i+1$. We wish to prove that a nondegenerate $S^{2 i} \mathrm{C}$ $p^{2 i+1}$ of index $i$ is a quadric. By 2.4.5, this assertion is true for $i=1$. We assume that it has been proven up to $i-1$.

Let $\left\{p_{\sigma}, q_{\sigma} \mid \sigma \in I\right\} \subset S^{n-1}$ satisfy 2.6.3. Then $R^{s}=\varnothing(2.6 .4(2))$ and by 2.6.5, the $i$-flats $\widetilde{P}^{i}, \widetilde{Q}^{i}, P_{\alpha}^{i}$, and $Q_{\alpha}^{i}$ are contained in $S^{n-1} ; \alpha \in I$. Finally, 2.6 .4 and $R^{s}=\varnothing$ yield

(1) $P^{n}=\left[p_{0}, \ldots, p_{i}, q_{0}, \ldots, q_{i}\right]=\left[\widetilde{P}^{i}, \widetilde{Q}^{i}\right]$,

(2) $\pi\left(p_{\alpha}\right)=\left[\tilde{P}^{i}, q_{0}, \ldots, \hat{q}_{\alpha}, \ldots, q_{i}\right], \alpha \in I$, and

(3) $\pi\left(q_{\alpha}\right)=\left[\widetilde{Q}^{i}, p_{0}, \ldots, \hat{p}_{\alpha}, \ldots, p_{i}\right], \alpha \in I$.

As $\widetilde{P}^{i} \cap \widetilde{Q}^{i}=\varnothing$, this implies that for $\alpha \neq \beta$ in $I$

(4) $\widetilde{P}^{i} \cap P_{\alpha}^{i}=\left[p_{0}, \ldots, \hat{p}_{\alpha}, \ldots, p_{i}\right]$ is an $(i-1)$-flat, and

(5) $P_{\alpha}^{i} \cap P_{\beta}^{i}=\left[p_{0}, \ldots, \hat{p}_{\alpha}, \ldots, \hat{p}_{\beta}, \ldots, p_{i}\right]$ is an $(i-2)$-flat.

Similarly, $\operatorname{dim}\left(\widetilde{Q}^{i} \cap Q_{\alpha}^{i}\right)=i-1$ and $\operatorname{dim}\left(Q_{\alpha}^{i} \cap Q_{\beta}^{i}\right)=i-2$.

A.4.2. Let $q_{0 \beta} \in\left[q_{0}, q_{\beta}\right] \backslash\left\{q_{0}, q_{\beta}\right\} ; \beta \in \Lambda\{0\}$. Then $q_{0 \beta} \notin \widetilde{P}^{i}$. As $P_{\alpha}^{i}=$ $\left[p_{0}, \ldots, \hat{p}_{\alpha}, \ldots, p_{i}, q_{\alpha}\right]$, this implies that for $\{\alpha, \beta\} \subset I, q_{0 \beta} \in\left[q_{0}, q_{\beta}\right] \subset$ 
$\bigcap_{\sigma=1 ; \sigma \neq \beta}^{i} \pi\left(p_{\sigma}\right)$. Thus by $2.2 .4, P_{0}^{i} \cap P_{\beta}^{i}=\left[p_{1}, \ldots, \hat{p}_{\beta}, \ldots, p_{i}\right] \subset \pi\left(q_{0 \beta}\right) \cap$ $S^{n-1}$ and $\left[q_{0 \beta}, P_{0}^{i} \cap P_{\beta}^{i}\right] \subset S^{n-1}$.

By 2.1.5, the $(i+1)$-section $\left[\widetilde{P}^{i}, q_{0 \beta}\right] \cap S^{n-1}$ is a pair of $i$-flats, $\widetilde{P}^{i}$ and say $P_{0 \beta}^{i}$. Clearly $q_{0 \beta} \in P_{0 \beta}^{i}$ and thus, $\left[q_{0 \beta}, P_{0}^{i} \cap P_{\beta}^{i}\right] \subset P_{0 \beta}^{i}$. Since $\operatorname{dim}\left(\widetilde{P}^{i} \cap P_{0 \beta}^{i}\right)=i-1$ and $\left[p_{0}, p_{\beta}\right] \cap\left(P_{0}^{i} \cap P_{\beta}^{i}\right)=\varnothing$, we obtain that $P_{0 \beta}^{i} \cap$ $\left[p_{0}, p_{\beta}\right]$ consists of a point, say $p_{0 \beta}$. Thus, $P_{0 \beta}^{i}=\left[q_{0 \beta}, p_{0 \beta}, P_{0}^{i} \cap P_{\beta}^{i}\right]$.

Since $\left[q_{0}, q_{\beta}\right] \cap \pi\left(p_{\beta}\right)=\left\{q_{0}\right\}$, we have $q_{0 \beta} \notin \pi\left(p_{\beta}\right)$. Then $P_{0 \beta}^{i} \not \subset \pi\left(p_{\beta}\right)$ and equivalently, $p_{\beta} \notin P_{0 \beta}^{i}$. Since $p_{0 \beta} \in P_{0 \beta}^{i}$, we have $p_{0 \beta} \neq p_{\beta}$. By a symmetric argument, $p_{0 \beta} \neq p_{0}$ as well.

In summary, $P_{0 \beta}^{i}=\left[q_{0 \beta}, p_{0 \beta}, P_{0}^{i} \cap P_{\beta}^{i}\right]$ where $q_{0 \beta} \in\left[q_{0}, q_{\beta}\right] \backslash\left\{q_{0}, q_{\beta}\right\}$ and $p_{0 \beta} \in\left[p_{0}, p_{\beta}\right] \backslash\left\{p_{0}, p_{\beta}\right\} ; \beta \in \Lambda\{0\}$. Clearly, $\pi\left(p_{0 \beta}\right)=\left[\widetilde{P}^{i}, q_{0 \beta}, q_{1}, \ldots, \hat{q}_{\beta}\right.$, $\left.\ldots, q_{i}\right]$.

A.4.3. LemmA. There is a unique nondegenerate $Q^{n-1} \subset P^{n}$ containing the i-flats $\widetilde{P}^{i}, \widetilde{Q}^{i}, P_{\alpha}^{i}$ and $P_{0 \beta}^{i},\{\alpha, \beta\} \subset I, \beta \neq 0 ; n=2 i+1 \geqslant 5$.

Proof. Choose the base points $u_{k}$ of $P^{n}$ as follows:

$$
u_{k}= \begin{cases}p_{k}, & k=0,1, \ldots, i, \\ q_{n-k}, & k=i+1, \ldots, n .\end{cases}
$$

Let $\beta \in \Lambda\{0\}$. Since $q_{0 \beta} \in\left[q_{0}, q_{\beta}\right] \backslash\left\{q_{0}, q_{\beta}\right\}$, let $q_{0 \beta} \equiv\left(0, \ldots, 0, x_{n-\beta}\right.$, $0, \ldots, 0,1), x_{n-\beta}=d_{\beta} \neq 0$. Then $p_{0 \beta} \in\left[p_{0}, p_{\beta}\right] \backslash\left\{p_{0}, p_{\beta}\right\}$ is determined and hence, $p_{0 \beta} \equiv\left(1,0, \ldots, 0, x_{\beta}, 0, \ldots, 0\right), x_{\beta}=c_{\beta} \neq 0$. Thus,

$$
\begin{array}{r}
P_{0 \beta}^{i} \equiv\left\{\left(x_{0}, \ldots, x_{i}, 0, \ldots, 0, x_{n-\beta}, 0, \ldots, 0, x_{n}\right) \mid\right. \\
\left.x_{\beta}=x_{0} c_{\beta} \text { and } x_{n-\beta}=x_{n} d_{\beta}\right\} .
\end{array}
$$

It is easy to verify that the quadric $Q^{n-1}$ given by

$$
\sum_{\sigma=1}^{i}\left(c_{\sigma} d_{\sigma}\right)^{-1} x_{\sigma} x_{n-\sigma}-x_{0} x_{n}=0
$$

satisfies A.4.3.

Corollary. $\omega\left(u_{\sigma}\right)=\pi\left(u_{\sigma}\right) ; \sigma=0,1, \ldots, n$.

Proof. By A.4.3(1), $\omega\left(u_{\sigma}\right) \equiv x_{n-\sigma}=0 ; \sigma=0,1, \ldots, n$. From A.4.1,

$$
\pi\left(u_{\sigma}\right)=\left[u_{0}, \ldots, \hat{u}_{n-\sigma}, \ldots, u_{n}\right] \equiv x_{n-\sigma}=0 ; \quad \sigma=0,1, \ldots, n \text {. }
$$

A.4.4. LemmA. $\pi\left(p_{\sigma}\right) \cap \pi\left(q_{\sigma}\right) \cap S^{n-1}=\pi\left(p_{\sigma}\right) \cap \pi\left(q_{\sigma}\right) \cap \mathrm{Q}^{n-1}$; $\sigma \in \Lambda\{0\}$

Proof. We may assume e.g. $\sigma=i$. From A.4.1, $P^{n-2}=\pi\left(p_{i}\right) \cap \pi\left(q_{i}\right)=$ 
$\left[p_{0}, \ldots, p_{i-1}, q_{0}, \ldots, q_{i-1}\right]$ is an $(n-2)$-flat and by $2.6 .2, P^{n-2} \cap S^{n-1}$ is a nondegenerate $S^{n-3}$; ind $S^{n-3}=i-1=1 / 2(n-3)$. As $n-2 \geqslant 3, S^{n-3}$ $C P^{n-2}$ is a quadric by the induction hypothesis.

The following $(i-1)$-flats are contained in $S^{n-3}: P^{n-2} \cap \widetilde{P}^{i}, P^{n-2} \cap$ $\widetilde{Q}^{i}, P^{n-2} \cap P_{\alpha}^{i}, \alpha \in \Lambda\{i\}$ and $P^{n-2} \cap P_{0 \beta}^{i}=\left[q_{0 \beta}, p_{0 \beta},\left(P^{n-2} \cap P_{0}^{i}\right) \cap\left(P^{n-2}\right.\right.$ $\left.\left.\cap P_{\beta}^{i}\right)\right], \beta \in \Lambda\{0, i\}$.

Using the coordinate system in A.4.3, $P^{n-2}$ is given by $x_{i}=x_{i+1}=0$. But then (cf. A.4.3) $S^{n-3}$ is defined by

$$
\sum_{\sigma=1}^{i-1}\left(c_{\sigma} d_{\sigma}\right)^{-1} x_{\sigma} x_{n-\sigma}-x_{0} x_{n}=0, \quad x_{i}=x_{i+1}=0 .
$$

By A.4.3(1), $S^{n-3}=P^{n-2} \cap Q^{n-1}$ and the lemma follows.

A.4.5. Lemma. $\pi\left(u_{\sigma}\right) \cap S^{n-1}=\pi\left(u_{\sigma}\right) \cap Q^{n-1} ; \sigma=0,1, \ldots, n$.

Proof. Recall that

$$
\pi\left(u_{\sigma}\right)=\omega\left(u_{\sigma}\right) \equiv x_{n-\sigma}=0 ; \quad \sigma=0,1, \ldots, n .
$$

For $0 \neq \sigma \neq n$, A.4.4 and (1) imply that $\pi\left(u_{\sigma}\right) \cap S^{n-1}$ and $\pi\left(u_{\sigma}\right) \cap$ $Q^{n-1}$ are cones with the vertex $u_{\sigma}$ and the $(n-2)$-section $\pi\left(u_{\sigma}\right) \cap \pi\left(u_{n-\sigma}\right) \cap$ $S^{n-1}$ and thus equal.

Let $\sigma=0$. We first prove that $\pi\left(u_{0}\right) \cap Q^{n-1} \subseteq \pi\left(u_{0}\right) \cap S^{n-1}$. Let $Q^{i} \subset$ $\pi\left(u_{0}\right) \cap Q^{n-1}$ be an $i$-flat. By the preceding, we may assume that $\pi\left(u_{\sigma}\right) \cap Q^{i}$ is an $(i-1)$-flat in $S^{n-1} ; \sigma=1, \ldots, n-1$. By 2.1.5, $Q^{i} \subset S^{n-1}$ if

$$
\left|\left\{\pi\left(u_{\sigma}\right) \cap Q^{i} \mid \sigma=1, \ldots, n-1\right\}\right| \geqslant 3 .
$$

Since $Q^{i} \subset \pi\left(u_{0}\right)$ is an $i$-flat and since $\pi\left(u_{0}\right)$ is given by $x_{n}=0,(1) \mathrm{im}$ plies that there are at least $i+1$ mutually distinct $\pi\left(u_{\sigma}\right) \cap Q$ 's for $\sigma=1,2$, $\ldots, n$. But then $u_{n} \notin Q^{i}$ and $i \geqslant 2$ imply (2). Therefore, $\pi\left(u_{0}\right) \cap Q^{n-1} \subseteq$ $\pi\left(u_{0}\right) \cap S^{n-1}$.

The preceding argument is symmetric in $\mathbf{Q}^{n-1}$ and $S^{n-1}$. Similarly, $\pi\left(u_{n}\right) \cap Q^{n-1}=\pi\left(u_{n}\right) \cap S^{n-1}$. $Q^{n-1}$.

A.4.6. THEOREM. Under the hypotheses of A.4.1 and A.4.3, $S^{n-1}=$

Proof. Cf. the proof of A.3.4.

A.5. Nondegenerate $S^{n-1} ; 1<$ ind $S^{n-1}<[1 / 2(n-1)]$.

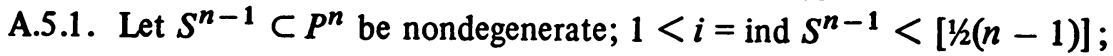
thus $n \geqslant 7$. Let $\left\{p_{\sigma}, q_{\sigma} \mid \sigma \in I\right\} \subset S^{n-1}$ satisfy 2.6.3. Let $m=n-2 i$. Then $R^{m}=\bigcap_{\sigma=0}^{i-1}\left(\pi\left(p_{\sigma}\right) \cap \pi\left(q_{\sigma}\right)\right)$ is an $m$-flat and $R^{m} \cap S^{n-1}$ is a nondegenerate $S^{m-1}$; ind $S^{m-1}=0$. By A.1.1, there is a $P^{m+1}$ through $R^{m}$ such that 
$P^{m+1} \cap S^{n-1}$ is a nondegenerate $S^{m}$; ind $S^{m}=1$. From A.2, $S^{m}$ and thus $S^{m-1}$ are quadrics.

Choose $m$ points $r_{\gamma} \in S^{m-1}$ such that $R^{m-1}=\left[r_{1}, \ldots, r_{m}\right] \subset R^{m}$ is an $(m-1)$-flat. As $S^{m-1}$ is a quadric, A.1.2 Corollary implies that $\bigcap_{\gamma=1}^{m}\left(\pi\left(r_{\gamma}\right) \cap R^{m}\right)$ is a point $r_{0} \notin R^{m-1}$. We assume $\left\{p_{i}, q_{i}\right\} \subset\left\{r_{1}, \ldots, r_{m}\right\}$ and thus, $r_{0} \notin S^{n-1}$ as

$$
\left\{r_{0}\right\}=\bigcap_{\gamma=1}^{m}\left(\pi\left(r_{\gamma}\right) \cap R^{m}\right) \subseteq \bigcap_{\sigma=0}^{i}\left(\pi\left(p_{\sigma}\right) \cap \pi\left(q_{\sigma}\right)\right)=R^{s} ; \text { cf. 2.6.5. }
$$

We apply 2.6 .7 in the case $k=i-1$ and $r=r_{0}$. Then $P^{n}=\left[R^{m}, P^{2 i}\right]$ where $R^{m}=\left[r_{0}, \ldots, r_{m}\right], P^{2 i}=\left[p_{0}, \ldots, p_{i-1}, r_{0}, q_{0}, \ldots, q_{i-1}\right], R^{m} \cap$ $P^{2 i}=\left\{r_{0}\right\}$ and $P^{2 i} \cap S^{n-1}$ is a nondegenerate $S^{2 i-1}$ with ind $S^{2 i-1}=i-1$.

We introduce the $i$-flats $\widetilde{P}^{i}, \widetilde{Q}^{i}, P_{\alpha}^{i}$ and $Q_{\alpha}^{i}$ in $S^{n-1}$ as in 2.6.4; $\alpha \in I$. Let $j=i-1$ and $J=\Lambda\{i\}$. Then the $j$-flats $\widetilde{P}^{j}=P^{2 i} \cap \widetilde{P}^{i}, \widetilde{Q}^{j}=P^{2 i} \cap \widetilde{Q}^{i}, P_{\alpha}^{j}=$ $P^{2 i} \cap P_{\alpha}^{i}$ and $Q_{\alpha}^{j}=P^{2 i} \cap Q_{\alpha}^{i}$ are contained in $S^{2 i-1} ; \alpha \in J$. Finally, $\left\{p_{i}, q_{i}\right\} \subset$ $\left\{r_{1}, \ldots, r_{m}\right\}$ implies $R^{m}=\left[R^{s}, p_{i}, q_{i}\right]$; (cf. the proof of 2.6 .3 Corollary). Then

$$
\begin{aligned}
\pi\left(p_{\alpha}\right) & =\left[p_{0}, \ldots, p_{i}, R^{s}, q_{0}, \ldots, \hat{q}_{\alpha}, \ldots, q_{i}\right] \\
& =\left[\widetilde{P}^{j}, R^{m}, q_{0}, \ldots, \hat{q}_{\alpha}, \ldots, q_{j}\right], \\
\pi\left(q_{\alpha}\right) & =\left[\widetilde{Q}^{j}, R^{m}, p_{0}, \ldots, \hat{p}_{\alpha}, \ldots, p_{j}\right], \alpha \in I ; \text { cf. 2.6.4. }
\end{aligned}
$$

A.5.2. LEMMA. (1) $P^{2 i} \subset \pi\left(r_{\gamma}\right) ; \gamma=1,2, \ldots, m$.

(2) $R^{m-1} \subset \pi(p)$ for each $p \in S^{2 i-1}$.

A.5.3. Let $u_{k}$ be the base points of $P^{n}$ where

$$
\begin{aligned}
u_{k} & =p_{k} ; \quad k=0,1, \ldots, i-1, \\
u_{i+k} & =r_{k} ; \quad k=0,1, \ldots, n-2 i, \\
u_{n-k} & =q_{k} ; \quad k=0,1, \ldots, i-1 .
\end{aligned}
$$

Let $Q^{n-1} \subset P^{n}$ be given by

$$
x_{i}^{2}+2 \sum_{\sigma=0}^{i-1} a_{\sigma, n-\sigma} x_{\sigma} x_{n-\sigma}+2 \sum_{\sigma, \mu=i+1 ; \sigma \neq \mu}^{n-i} a_{\sigma \mu} x_{\sigma} x_{\mu}=0 ; a_{\sigma \beta} \neq 0 \text {. }
$$

Then $r_{0} \notin Q^{n-1}$ and $Q^{n-1}$ contains $\widetilde{P}^{j}, \widetilde{Q}^{j}, P_{\alpha}^{j}$ and $Q_{\alpha}^{j} ; \alpha \in J$.

We have observed that $S^{m-1}$ is a quadric. As ind $S^{2 i-1}=[1 / 2(2 i-1)]$, $S^{2 i-1}$ is a quadric from A.3. Thus we can choose $u_{\sigma \beta}$ in (1) so that $S^{m-1}=$ $R^{m} \cap Q^{n-1}$ and $S^{2 i-1}=P^{2 i} \cap Q^{n-1}$. This determines $Q^{n-1}$ uniquely.

Let $b \equiv\left(b_{0}, \ldots, b_{n}\right) \in S^{2 i-1}$. Then $b_{i+1}=\cdots=b_{n-i}=0$ and $\omega(b) \equiv b_{i} x_{i}+\Sigma_{\sigma=0}^{i-1} a_{\sigma, n-\sigma}\left(b_{\sigma} x_{n-\sigma}+b_{n-\sigma} x_{\sigma}\right)=0$. Also for $\sigma-1, \ldots, m$, $\omega\left(r_{\sigma}\right)=\omega\left(u_{i+\sigma}\right) \equiv \sum_{\mu=i+1 ; \mu \neq \sigma}^{n-i} a_{\sigma \mu} x_{\mu}=0$.

Clearly, $P^{2 i} \subset \omega\left(r_{\sigma}\right)$ for $\sigma=1, \ldots, m$ and $R^{m-1} \subset \omega(p)$ for each 
$p \in S^{2 i-1}$. Finally, $\omega\left(p_{\sigma}\right)$ is the hyperplane given by $x_{n-\sigma}=0$. Thus,

$$
\omega\left(p_{\sigma}\right)=\left[p_{0}, \ldots, p_{i-1}, r_{0}, \ldots, r_{m}, q_{0}, \ldots, \hat{q}_{\sigma}, \ldots, q_{i-1}\right]=\pi\left(p_{\sigma}\right) .
$$

Similarly, $\omega\left(q_{\sigma}\right)=\pi\left(q_{\sigma}\right)$ is given by $x_{\sigma}=0 ; \sigma \in J$. Hence, $\left\{u_{i}\right\}=\left\{r_{0}\right\} \subseteq$ $\cap_{\sigma=0 ; \sigma \neq i}^{n} \omega\left(u_{\sigma}\right)$.

A.5.4. We have shown that a nondegenerate $S^{n-1} \subset P^{n}$ with ind $S^{n-1}=1$ or $[1 / 2(n-1)]$ is a quadric; $n \geqslant 3$. In particular, every nondegenerate $S^{n-1}$ with ind $S^{n-1}>0$ is a quadric when $n=3,4,5$ or 6 . Since $n \geqslant 7$, we assume every nondegenerate $S^{k-1} \subset P^{k}$, with ind $S^{k-1}>0$ and $k<n$ is a quadric.

A.5.5. LемMA. $\pi\left(p_{\sigma}\right) \cap \pi\left(q_{\sigma}\right) \cap S^{n-1}=\pi\left(p_{\sigma}\right) \cap \pi\left(q_{\sigma}\right) \cap Q^{n-1} ; \sigma \in J$.

Proof. By the symmetry in $\sigma \in J$, we may assume $\sigma=i-1$. Now

$$
P^{n-2}=\pi\left(p_{i-1}\right) \cap \pi\left(q_{i-1}\right)=\left[p_{0}, \ldots, p_{i-2}, R^{m}, q_{0}, \ldots, q_{i-2}\right]
$$

is an $(n-2)$-flat. By 2.6.2, $P^{n-2} \cap S^{n-1}$ is a nondegenerate $S^{n-3}$; ind $S^{n-3}$ $=i-1$. By A.5.4, $S^{n-3}$ is a quadric $Q^{n-3}$.

Clearly, $S^{m-1} \subset S^{n-3}$ and $S^{n-3}$ contains the $(i-2)$-flats $P^{n-2} \cap \widetilde{P}^{j}$, $P^{n-2} \cap \widetilde{Q}^{j}, P^{n-2} \cap P_{\alpha}^{j}$ and $P^{n-2} \cap Q_{\alpha}^{j} ; \alpha=0,1, \ldots, i-2$.

Using the base points in A.5.3, $P^{n-2}$ is given by $x_{i-1}=x_{n-i+1}=0$. Let $Q^{n-3}$ be given by

$$
x_{i}^{2}+2 \sum_{\sigma=0}^{i-2} d_{\sigma, n-\sigma} x_{\sigma} x_{n-\sigma}+2 \sum_{\sigma, \mu=i+1 ; \sigma \neq \mu}^{n-i} d_{\sigma \mu} x_{\sigma} x_{\mu}=0, \quad x_{i-1}=x_{n-i+1}=0 \text {. }
$$

Then $S^{m-1}=R^{m} \cap Q^{n-1}=R^{m} \cap Q^{n-3}$ implies that $a_{\sigma \mu}=d_{\sigma \mu} ; \sigma \neq \mu, \sigma, \mu=$ $i+1, \ldots, n-i$. Similarly (cf. the proof of A.3.3), $S_{\mu}^{1}=P_{\mu}^{2} \cap Q^{n-1}=P_{\mu}^{2} \cap$ $\mathrm{Q}^{n-3}$ implies that $a_{\mu, n-\mu}=d_{\mu, n-\mu} ; \mu=0,1, \ldots, i-2$. Thus $\mathbf{Q}^{n-3} \subset \mathrm{Q}^{n-1}$ and $P^{n-2} \cap S^{n-1}=S^{n-3}=Q^{n-3}=P^{n-2} \cap Q^{n-1}$.

COROllaRY. $\pi\left(u_{\sigma}\right) \cap S^{n-1}=\pi\left(u_{\sigma}\right) \cap Q^{n-1} ; \sigma=0, \ldots, i-1$, $n-i+1, \ldots, n$.

\section{A.5.6. Lemma. Let $p \in S^{2 i-1} \cup S^{m-1}$. Then $\pi(p)=\omega(p)$.}

Proof. Let $\pi^{\prime}(p)[\tilde{\pi}(p)]$ be the tangent hyperplane of $S^{2 i-1}\left[S^{m-1}\right]$ at a point $p$. Then $\operatorname{dim} \pi^{\prime}(p)=2 i-1$ and $\operatorname{dim} \tilde{\pi}(p)=m-1$.

If $p \in S^{2 i-1}$, then clearly $\pi(p)=\left[\pi^{\prime}(p), R^{m-1}\right]$. Since $S^{2 i-1}=P^{2 i} \cap$ $Q^{n-1}$, we have $\pi^{\prime}(p) \subseteq \omega(p)$ and thus, $\omega(p)=\left[\pi^{\prime}(p), R^{m-1}\right]$.

If $p \in S^{m-1}=R^{m} \cap Q^{n-1}$, then $\tilde{\pi}(p) \subseteq \pi(p) \cap \omega(p)$. From the construction, $\left[\widetilde{P}^{i-1}, \widetilde{Q}^{i-1}\right] \subseteq \pi(p) \cap \omega(p)$ and thus, $\left[\tilde{\pi}(p), \widetilde{P}^{i-1}, \widetilde{Q}^{i-1}\right] \subseteq \pi(p)$ $\cap \omega(p)$. But $\tilde{\pi}(p) \subset R^{m}$ and $R^{m} \cap\left[\widetilde{P}^{i-1}, \widetilde{Q}^{i-1}\right]=\varnothing$ imply

$$
\operatorname{dim}\left[\tilde{\pi}(p), \widetilde{P}^{i-1}, \widetilde{Q}^{i-1}\right]=n-1 .
$$
$\mathbf{Q}^{n-1}$.

A.5.7. Lemma. Let $p \in S^{2 i-1} \cup S^{m-1}$. Then $\pi(p) \cap S^{n-1}=\pi(p) \cap$ 
Proof. By A.5.6, it is sufficient to find a $P^{n-2} \subset \pi(p)$ such that $\pi(p)=$ $\left[P^{n-2}, p\right]$ and $P^{n-2} \cap S^{n-1}=P^{n-2} \cap Q^{n-1}$; for then, $\pi(p) \cap S^{n-1}$ and $\pi(p) \cap Q^{n-1}$ are cones with the vertex $p$ and the same $(n-2)$-section.

Let $p \in S^{2 i-1}$. Since $P^{2 i} \cap R^{m}=\left\{r_{0}\right\} \not \subset S^{n-1}$, we have $p \notin R^{m}$ and thus, $p \notin \pi\left(p_{0}\right)$ say. Take $P^{n-2}=\pi(p) \cap \pi\left(p_{0}\right)$. Then by A.5.5 Corollary,

$$
\begin{aligned}
P^{n-2} \cap S^{n-1} & =P^{n-2} \cap\left(\pi\left(p_{0}\right) \cap S^{n-1}\right)=P^{n-2} \cap\left(\pi\left(p_{0}\right) \cap Q^{n-1}\right) \\
& =P^{n-2} \cap Q^{n-1}
\end{aligned}
$$

Let $p \in S^{m-1}$ and let $P^{2}=\left[p_{0}, q_{0}, r_{0}\right]$. Obviously, $P^{2} \cap S^{n-1}$ is an $S^{1} \subset S^{2 i-1}$. Now $r_{0} \in \pi\left(p_{0}\right) \cap \pi\left(q_{0}\right)$ implies that $r_{0} \notin \pi(q)$ for each $q \in$ $S^{1} \backslash\left\{p_{0}, q_{0}\right\}$. By A.5.2, $R^{m-1}=\pi(q) \cap R^{m}$ for each $q \in S^{1} \backslash\left\{p_{0}, q_{0}\right\}$.

If $p \in S^{m-1} \backslash R^{m-1}$, then $p \notin \pi(q)$ for some $q \in S^{1} \subset S^{2 i-1}$. By the preceding, $\pi(p) \cap\left(\pi(q) \cap S^{n-1}\right)=\pi(p) \cap\left(\pi(q) \cap Q^{n-1}\right)$.

If $p \in R^{m-1} \cap S^{m-1}$, then ind $S^{m-1}=0$ implies that $p \notin \pi\left(p^{\prime}\right)$ for any $p^{\prime} \in S^{m-1} \backslash R^{m-1}$. The lemma now follows as above.

A.5.8. Let $M=\left\{u_{0}, \ldots, u_{i-1}, u_{n-i+1}, \ldots, u_{n}\right\}$. From A.5.3, $\pi\left(u_{\sigma}\right)=$ $\omega\left(u_{\sigma}\right)$ is given by $x_{n-\sigma}=0$ for each $u_{\sigma} \in M$. Thus for $\left\{u_{\sigma_{1}}, \ldots, u_{\sigma_{k}}\right\} \subset M$, $P^{n-k}=\bigcap_{j=1}^{k} \pi\left(u_{\sigma_{j}}\right)$ is an $(n-k)$-flat.

Let $M^{i}$ be an $i$-flat; $M^{i} \cap R^{m}=\varnothing$. Assume that $M_{\sigma}^{i-1}=\pi\left(u_{\sigma}\right) \cap M^{i}$ is an (i-1)-flat for each $u_{\sigma} \in M$. Let $U=\left\{M_{\sigma}^{i-1} \mid u_{\sigma} \in M\right\}$.

A.5.9. LemMA. $M^{i-1}=M_{\sigma}^{i-1}$ has at most $i$ solutions $u_{\sigma}$ in $M$.

Proof. Let $\left\{u_{\sigma_{1}}, \ldots, u_{\sigma_{k}}\right\} \subset M$ be the set of solutions of $M^{i-1}=$ $M_{\sigma}^{i-1}$. Then $M^{i-1}=\bigcap_{j=1}^{k} M_{\sigma_{j}}^{i-1}=\left(\bigcap_{j=1}^{k} \pi\left(u_{\sigma_{j}}\right)\right) \cap M^{i}=P^{n-k} \cap M^{i}$. As $R^{m}=\bigcap_{u_{\sigma} \in \pi} \pi\left(u_{\sigma}\right)$, this implies that $\left[R^{m}, M^{i-1}\right] \subseteq P^{n-k}$. But $R^{m} \cap M^{i}=\varnothing$ implies that $\operatorname{dim}\left(\left[R^{m}, M^{i-1}\right]\right)=n-i$ and thus, $k \leqslant i$.

COROllary 1. Let $M^{i-1}=M_{\sigma}^{i-1}\left[\bar{M}^{i-1}=M_{\sigma}^{i-1}\right]$ have $k[h]$ solutions in $M ; M^{i-1} \neq \bar{M}^{i-1}$. Then $k+h \leqslant i+1$.

Proof. Let $\left\{u_{\sigma_{1}}, \ldots, u_{\sigma_{k}}\right\}\left[\left\{u_{\sigma_{k+1}}, \ldots, u_{\sigma_{k+h}}\right\}\right]$ be the two sets of solutions in $M$. Then

$$
M^{i-1}=\left(\bigcap_{j=1}^{k} \pi\left(u_{\sigma_{j}}\right)\right) \cap M^{i} \text { and } \bar{M}^{i-1}=\left(\bigcap_{j=k+1}^{k+h} \pi\left(u_{\sigma_{j}}\right)\right) \cap M^{i} \text {. }
$$

Since $M^{i}=\left[M^{i-1}, \bar{M}^{i-1}\right], M^{i-2}=M^{i-1} \cap \bar{M}^{i-1}$ is an $(i-2)$-flat, and $M^{i-2}=\left(\bigcap_{j=1}^{k+h} \pi\left(u_{\sigma_{j}}\right)\right) \cap M^{i}=P^{n-(k+h)} \cap M^{i}$. As in A.5.9, $\left[R^{m}, M^{i-2}\right] \subseteq$ $p^{n-k-h}$ and $k+h \leqslant i+1$.

Corollary 2. $|U| \geqslant 3$. 
Proof. As $|M|=2 i$, A.5.9 implies that $|U| \geqslant 2$. Since $i \geqslant 2$, we have $|M|=2 i>i+1$ and by Corollary $1,|U| \geqslant 3$.

A.5.10. THEOREM. Under the hypotheses of A.5.1 and A.5.3, $S^{n-1}=$ $\mathbf{Q}^{n-1}$.

Proof. We first prove $Q^{n-1} \subseteq S^{n-1}$. Let the $i$-flat $M^{i} \subset Q^{n-1}$. By A.5.6 and A.5.7, we may assume that $M^{i} \cap R^{m}=M^{i} \cap P^{2 i}=\varnothing$. Since $M \subset$ $P^{2 i} ; u_{\sigma} \notin M^{i}$ and thus, $M_{\sigma}^{i-1}=\pi\left(u_{\sigma}\right) \cap M^{i} \subset S^{n-1}$ is an $(i-1)$-flat for each $u_{\sigma} \in M$. By A.5.9 Corollary $2,|U| \geqslant 3$ and thus, $M^{i} \subset S^{n-1}$ by 2.1.5.

The preceding argument is symmetric in $S^{n-1}$ and $Q^{n-1}$.

We collect our results.

A.5.11. THEOREM. A nondegenerate $S^{n-1}$ with ind $S^{n-1}>0$ is a quadric; $n \geqslant 3$.

\section{BIBLIOGRAPHY}

1. O. Haupt and H. Künneth, Geometrische Ordnungen, Die Grundlehren der math. Wissenschaften, Band 133, Springer-Verlag, Berlin and New York, 1967. MR 37 \#3491.

2. A. Marchaud, Les surfaces du second ordre en géométrie finie, J. Math. Pures Appl. 15 (1936), 293-300.

3. - Sur les ensembles linéairement connexes, Ann. Mat. Pura Appl. (4) 56 (1961), 131-157. MR 30 \#487.

4. R. Park, Topics in direct differential geometry, Canad. J. Math. 24 (1972), 98148. MR 45 \#2640.

5. P. Scherk, Über differenzierbare Kurven und Bögen. 1. Zum Begriff der Charakteristik, Časopis Pěst. Mat. Fys. 66 (1937), 165-171.

DEPARTMENT OF MATHEMATICS, UNIVERSITY OF TORONTO, TORONTO (181) ONTARIO, CANADA

Current address: Department of Mathematics and Statistics, The University of Calgary, Calgary 44, Alberta, Canada 